\title{
mTOR/Raptor signaling is critical for skeletogenesis in mice through the regulation of Runx2 expression
}

\author{
Qinggang Dai ${ }^{1,2}$, Zhan $\mathrm{Xu}^{2}$, Xuhui $\mathrm{Ma}^{3}$, Ningning Niư ${ }^{2}$, Siru Zhou ${ }^{4}$, Furong Xie ${ }^{1}$, Lingyong Jiang ${ }^{4}$, Jun Wang ${ }^{\star, 1}$ and Weiguo Zou ${ }^{\star, 2}$
}

The mammalian target of rapamycin (mTOR)/regulatory-associated protein of mTOR (Raptor) pathway transmits and integrates different signals including growth factors, nutrients, and energy metabolism. Nearly all these signals have been found to play roles in skeletal biology. However, the contribution of mTOR/Raptor to osteoblast biology in vivo remains to be elucidated as the conclusions of recent studies are controversial. Here we report that mice with a deficiency of either mTOR or Raptor in preosteoblasts exhibited clavicular hypoplasia and delayed fontanelle fusion, similar to those found in human patients with cleidocranial dysplasia (CCD) haploinsufficient for the transcription factor runt-related transcription factor 2 (Runx2) or those identified in Runx2 ${ }^{+-}$mice. Mechanistic analysis revealed that the mTOR-Raptor-S6K1 axis regulates Runx2 expression through phosphorylation of estrogen receptor $\alpha$, which binds to Distal-less homeobox 5 (DLX5) and augments the activity of Runx2 enhancer. Moreover, heterozygous mutation of raptor in osteoblasts aggravates the bone defects observed in Runx2 ${ }^{+/-}$mice, indicating a genetic interaction between Raptor and Runx2. Collectively, these findings reveal that mTOR/Raptor signaling is essential for bone formation in vivo through the regulation of Runx2 expression. These results also suggest that a selective mTOR/ Raptor antagonist, which has been developed for treatment of many diseases, may have the side effect of causing bone loss. Cell Death and Differentiation (2017) 24, 1886-1899; doi:10.1038/cdd.2017.110; published online 7 July 2017

Osteoblasts, the bone-forming cells active during bone development and remodeling, ${ }^{1,2}$ are derived from bone marrow mesenchymal stem cells (BMSCs). The differentiation of osteoblasts from BMSCs is controlled by transcription factors that are expressed in a defined temporal and spatial sequence. Among them, runt-related transcription factor 2 (Runx2) is considered to be the master transcription factor ${ }^{3,4}$ as mice deficient in Runx2 exhibit a lack of mineralization in the skeleton and absence of mature osteoblasts. ${ }^{5}$ Runx2heterozygous mice display clavicular hypoplasia and delayed closure of the fontanelles, a phenotype resembling the cleidocranial dysplasia (CCD) syndrome caused by mutations of Runx2 in humans. ${ }^{6}$ Runx 2 expression at appropriate times and sites is essential for bone development and bone remodeling. However, regulation of Runx2 expression, especially the upstream signaling pathways involved, has not been completely clarified.

The mammalian/mechanistic target of rapamycin (mTOR) is an evolutionarily conserved protein kinase. mTOR functions in two structurally and functionally distinct multiprotein complexes, namely mTORC 1 and $\mathrm{mTORC} 2,{ }^{7}$ which are distinct in their unique components and downstream targets. mTORC1 contains Raptor and is sensitive to rapamycin, while mTORC2 contains Rictor and is resistant to rapamycin. S6 kinase1
(S6K1) is the mTORC1 downstream target and can phosphorylate a series of substrates including estrogen receptor alpha (ERa) and S6 ribosomal protein (S6) to control gene transcription, protein synthesis, and other biological processes. $^{8-10}$ mTORC1 signaling is considered as the check-point of several extracellular and intracellular signals including growth factors, nutrients, energy metabolism and stress ${ }^{11}$ and has been the target for drug development in many diseases, ${ }^{11}$ which highlights the necessity of studying the role of mTORC1 in osteoblasts and bone development to monitor and avoid possible side effects on bone.

Recently, different studies of the role of mTORC1 signaling in osteoblast differentiation and bone development produced controversial results. ${ }^{12}$ Previous in vitro studies shown that mTORC1 can activate ${ }^{13,14}$ or inhibit ${ }^{15}$ osteogenesis, and these controversies may be resulted from the differences in the cell types or cell differentiation-stages examined. Furthermore, disturbing of mTOR signaling in osteoblast-lineage cells induced various skeletal disorders. A mouse model with increased $\mathrm{mTORC} 1$ activity in neural crest-derived cells due to deletion of tuberous sclerosis 1 (Tsc1) led to increased bone mass through enlargement of the osteoprogenitor pool. ${ }^{16}$ Interesting, two independent studies indicated that osteoblastspecific inactivation of Tsc complex caused osteoblasts to

${ }^{1}$ Department of Pediatric Dentistry, Shanghai Ninth People's Hospital, School of Medicine, Shanghai Jiaotong University; Shanghai Key Laboratory of Stomatology, Shanghai 200011, China; ${ }^{2}$ State Key Laboratory of Cell Biology, CAS Center for Excellence in Molecular Cell Science, Shanghai Institute of Biochemistry and Cell Biology, Chinese Academy of Sciences; University of Chinese Academy of Sciences, Shanghai 200031, China; ${ }^{3}$ Department of Oral and Maxillofacial-Head and Neck Oncology, Ninth People's Hospital, Shanghai Jiaotong University School of Medicine, Shanghai Key Laboratory of Stomatology, Shanghai 200011, China and ${ }^{4}$ Center of Craniofacial Orthodontics, Department of Oral and Cranio-Maxillofacial Science, Ninth People's Hospital, Shanghai Jiaotong University School of Medicine, Shanghai Key Laboratory of Stomatology, Shanghai 200011, China

${ }^{*}$ Corresponding author: J Wang, Department of Pediatric Dentistry, Shanghai Ninth People's Hospital, School of Medicine, Shanghai Jiaotong University, No. 639, Zhi Zao Ju Road, Shanghai 200011, China. Tel: +86 21 23271699; Fax: +86 21 23271699; E-mail: wangjun202@ 126.com

or W Zou, Shanghai Institute of Biochemistry and Cell Biology, Chinese Academy of Sciences; University of Chinese Academy of Sciences, 320 Yueyang Road, SIBCB Building, Rm 1401, Shanghai 200031, China. Tel: +86 21 54921320; Fax: +86 21 54921320; E-mail: zouwg94@ sibcb.ac.cn

Received 07.2.17; revised 08.5.17; accepted 31.5.17; Edited by M Piacentini; published online 07.7.17 
differentiate poorly and produce disorganized bone. ${ }^{17,18}$ On the other hand, Chen et al. reported that enhanced mTORC1 signaling due to heterozygous mutations in the fibrillin-1 gene resulted in osteopenia. ${ }^{19}$ Furthermore, decreased mTORC1 signaling with deletion of mTOR or Raptor in mesenchyme resulted in death shortly after birth and skeletal discrepancy. ${ }^{20}$ Raptor deletion in Osterix-expressing preosteoblasts led to osteopenia. $^{21}$ In contrast, an in vitro study showed that depletion of Raptor promoted osteoblast differentiation of BMSCs. $^{22}$ Taken together, these data reveal that the role of mTORC1 signaling in osteoblasts is still ambiguous and the underlying mechanisms have not been fully illuminated.

In the present study, we found that loss of mTORC1 signaling in preosteoblasts through the deletion of $m$ TOR or Raptor in mice induced severe skeletal defects secondary to impaired osteogenesis and osteoblast differentiation. Further molecular mechanism studies revealed that the mTORRaptor-S6K1 axis could promote osteoblast differentiation through regulating Runx2 expression by augmenting the activity of Runx2 enhancer.

\section{Results}

mTOR deficiency in preosteoblasts causes a CCD phenotype with impaired osteoblast differentiation. To determine the role of mTOR signaling in preosteoblasts in vivo, we generated conditional mTOR knockout mice

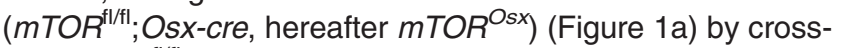
ing $m T O R^{f l / f l}$ mice with Osx-cre mice, a transgenic line in which Cre activation is confined to osteoblast precursors. Western blot assay confirmed the loss of mTOR protein in parietal bones from $m$ TOR ${ }^{O s x}$ newborns (Figure 1b). Compared to WT mice and mice heterozygous for the mTOR floxed allele $\left(m T O R^{f /++} ; O s x\right.$-cre, hereafter $\left.m T O R^{O s x /+}\right)$, $m T O R^{O s x}$ mice showed slower growth rate (Figures 1c and d). Alizarin red and Alcian blue staining showed although newborn $m T O R^{O s x}$ mice had similar skeletal size to their WT littermates (Figure 1e), mTOR ${ }^{O s x}$ mice exhibited the CCD phenotype with clavicular hypoplasia and hypomineralization of the calvarium (Figure 1f), features of mice heterozygous for Runx2.

In addition to the clavicular and calvarial phenotypes, $m T O R^{O s x}$ mice displayed a substantial reduction in bone mass. As shown in Figures $2 \mathrm{a}$ and $\mathrm{b}$, femoral trabecular bone of 4-week-old male $m T O R^{O s x}$ mice displayed an approximately $70 \%$ reduction in bone volume fraction (BV/TV) compared to WT littermates while $m T O R^{O s x /+}$ showed an intermediate effect (Figure $2 \mathrm{~b}$ ). The significant reduction in bone mass between $m T O R^{O s x}$ and $m T O R^{O s x /+}$ confirmed that the decreased bone density in $m T O R^{O s x}$ mice is due to the loss of $m T O R$ in osteoblasts, not to the presence of the Cre transgene. $m T O R^{O s x}$ mice also displayed a decrease of trabecular number (Tb.N., Figure 2c) and trabecular thickness (Tb.Th., Figure 2d). mTOR ${ }^{\text {osx }}$ mice also displayed a dramatic reduction of the cortical thickness (Ct.Th., Figure 2e).

We next examined whether the abnormal osteogenesis in $m T O R^{o s x}$ mice was a result of inadequate osteoblast differentiation. We cultured calvarial cells from $m T O R^{O s x}$ and WT mice and found that $m T O R^{O s x}$ calvarial cells showed reduced osteoblast differentiation, revealed by decreased alkaline phosphatase (ALP) activity and fewer calcified nodules, measured by alizarin red staining (Figure 2f). Consistent with the reduced ossification and osteoblast differentiation, expression of the characteristic osteoblast marker genes, collagen $1 a 1$ (Col1a 1, Figures $2 \mathrm{i}$ and $\mathrm{j}$ ) and osteocalcin (Ocn, Figures $2 \mathrm{i}$ and $\mathrm{k}$ ) were reduced in osteoblasts from $m T O R^{o s x}$ mice in vivo accompanied with the decrease of Runx2 expression (Figures $2 \mathrm{~g}$ and $\mathrm{h}$ ). Taken together, the CCD phenotype and reduced bone mass, possibly secondary to impaired osteoblastic differentiation in $m T O R^{o s x}$ mice, supported the hypothesis that mTOR is critical for bone development.

Raptor deficiency in preosteoblasts also causes a CCD phenotype and reduced bone mass with impaired osteoblast differentiation. To further investigate the specific contribution of mTORC1 in osteoblasts, we generated conditional Raptor knockout mice $\left(\operatorname{Rap}^{f / / f l} ;\right.$ Osx-cre, hereafter $\operatorname{Rap}^{O s x}$ ) by mating $\operatorname{Rap}^{f / / f l}$ mice with Osx-cre mice (Figure 3a). Western blot assay revealed the loss of Raptor protein in parietal bones from $\operatorname{Rap}^{O s x}$ mice (Figure $3 b$ ). Similar to mTOR ${ }^{\text {osx }}$ mice, Rap $^{\text {osx }}$ mice showed a slower growth pattern after birth (Figures $3 \mathrm{c}$ and d). The newborn Rap ${ }^{O s x}$ mice also exhibited the CCD phenotype with clavicular hypoplasia and hypomineralization of the calvarium (Figure $3 f$ ) although Rap $^{\text {Osx }}$ mice had similar skeletal size to their WT littermates (Figure 3e). Hypocalcification of calvarial and clavical bones were confirmed in 4-week-old $\operatorname{Rap}^{O s x}$ mice by micro-CT and X-ray analysis (Figure $3 \mathrm{~g}$ ). Moreover, 4-week-old Rap Osx mice had an osteopenic phenotype with decreased BV/TV, Tb.N., Tb.Th. and Ct.Th. in the femur (Figures $4 a-e)$. Further, 6-month-old Rap ${ }^{\text {Sx }}$ mice showed CCD phenotype with clavicular hypoplasia and hypomineralization of the calvarium (Supplementary Figure 1A). Micro-CT scanning confirmed calvarial hypoplasia (Supplementary Figure 1B) and showed deceased BV/TV, Tb.N., Tb.Th. and Ct.Th. in Rap ${ }^{\text {sx }}$ mice in comparison with WT mice (Supplementary Figure 1C-G). The significant difference between $\operatorname{Rap}^{O s x}$ mice and Rap ${ }^{O s x /+}$ mice accompanied with the skeletal defects in 6-month-old Rap ${ }^{\text {Osx }}$ mice confirmed that it is the loss of Raptor in osteoblasts, not the presence of the Cre transgene, that is responsible for the bone defects in $\operatorname{Rap}^{O s x}$ mice.

Primary osteoblast cultures from $\operatorname{Rap}^{\text {Osx }}$ mice exhibited decreased osteoblast differentiation, determined by decreased ALP activity and mineralized matrix production (Figure 4f). Consistent with this, $\operatorname{Rap}^{O s x}$ mice displayed decreased Col1a 1 and Ocn mRNA expression in vivo (Figures 4i-k) as well as reduced Runx2 protein expression (Figures $4 \mathrm{~g}$ and $\mathrm{h}$ ). The similar bone phenotype between Rap ${ }^{\text {sx }}$ mice and $m T O R^{O s x}$ mice supported the hypothesis that mTOR drives skeletal development mainly via the mTORC1 complex. The decreased bone mass in both $m T O R^{O s x}$ and Rap ${ }^{\text {sx }}$ mice might arise through increased bone resorption. To address this, we analyzed femurs from 4-week-old mice for the presence of tartrate-resistant acid phosphatase (TRAP)positive osteoclasts. In comparison with WT littermates, both $m T O R^{O s x}$ and $\operatorname{Rap}^{O s x}$ mice displayed a modest decrease in TRAP-positive osteoclasts (Supplementary Figures 2A and $\mathrm{B}$ ), indicating that the bone lesions in $m T O R^{O s x}$ or Rap ${ }^{\text {sx }}$ mice are attributable to impaired bone formation as opposed to 
a

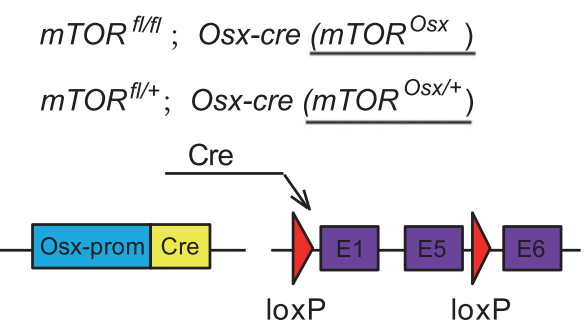

C

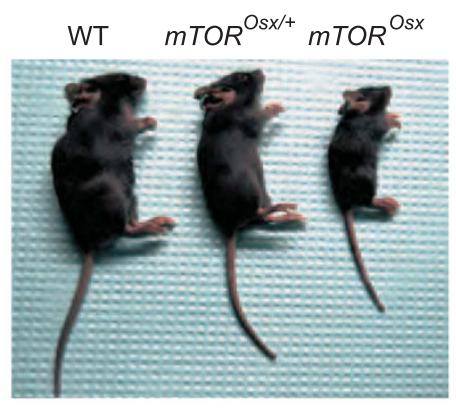

e

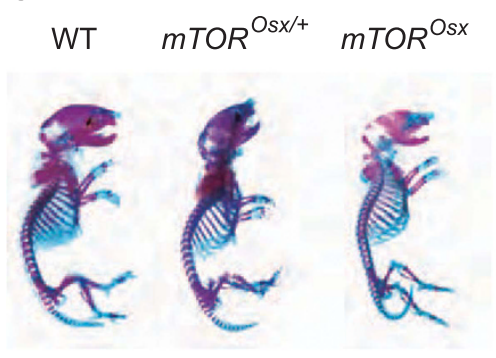

b

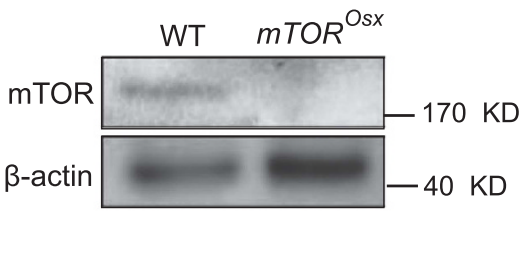

d

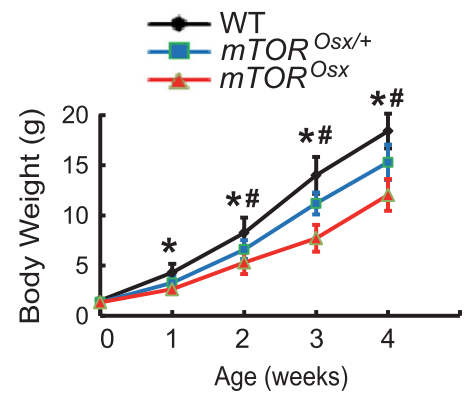

f

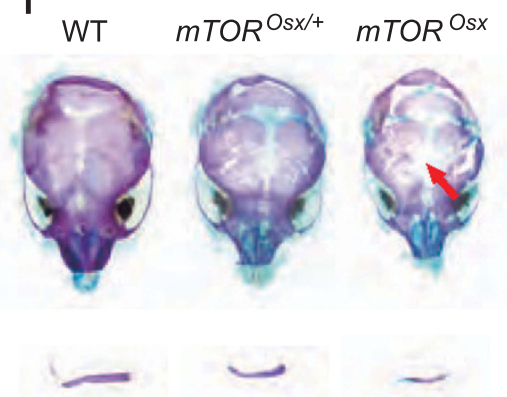

Figure 1 Preosteoblast-specific knockout of $m T O R$-induced CCD-like bone defects in mice. (a) Illustration of $m$ TOR deletion in Osx-expressing preosteoblasts. (b) Western blot assay of mTOR in parietal bone lysate of WT and $m T O R^{O s x}$ newborns. (c) Representative view of 4-week-old WT, $m T O R^{O s x /+}$ and $m T O R^{O s x}$ mice. (d) Body weight of male WT, $m T O R^{O s x /+}$ and $m T O R^{O s x}$ littermates measured at different age points $\left(n=5\right.$ for each group, $t$-test). Data represent means $\pm s$.d. ${ }^{*} P<0.05$ in comparison with WT mice, ${ }^{\#} P<0.05$ when compared with $m T O R^{O s x /+}$ mice. (e) Representative images of skeleton of WT, $m T O R^{O s x /+}$ and $m T O R^{O s x}$ newborn littermates by alcian blue and alizarin red staining. (f) Clavicular hypoplasia and calvarial hypomineralization of $m T O R^{O s x}$ newborns. Arrow indicates calvarial lesions in $m T O R^{O s x}$ mice

decreased bone resorption. Further confirming the decrease in osteoblast activity, 4-week-old $\operatorname{Rap}^{\text {Osx }}$ mice demonstrated a decreased bone formation rate (BFR) and mineral apposition rate (MAR) as determined by alizarin red and calcein labeling (Figures $4 \mathrm{l}$ and $\mathrm{m}$ ). Collectively, these data support the hypothesis that mTOR/Raptor (mTORC1) is critical for osteoblast activity and anabolic bone formation.

S6K1 is a downstream factor of mTOR/Raptor in osteoblasts. S6K1 is the most important downstream regulator of $\mathrm{mTORC1}$ and plays crucial roles in development and aging. To determine whether S6K1 could function downstream of mTOR/Raptor in osteoblasts, we analyzed the level of phospho-S6K1in 7-day-old mice by immunohistochemistry. An antibody specific to phospho-S6K1 (T389) demonstrated robust signal in osteoblast-lining cells in subchondral trabecular bone and in adjacent osteocytes (Figures $5 \mathrm{a}$ and $\mathrm{d}$ ). At the same time, both $m T O R^{O s x}$ and Rap $^{\text {Osx }}$ mice showed decreased S6K1 phosphorylation in comparison with their WT littermates (Figures 5a,b,d and e).
We also analyzed lysates from parietal bone of $m T O R^{O s x}$ and Rap $^{\text {Osx }}$ newborn mice. As shown in Figures $5 \mathrm{c}$ and $\mathrm{f}$, although the expression of S6K1 is comparable between WT mice and $m T O R^{O s x}$ or Rap ${ }^{\text {sx }}$ mice, the level of phosphorylation of S6K1 in mTOR ${ }^{O s x}$ or Rap ${ }^{\text {Sx }}$ mice was dramatically decreased. To examined whether the reduction of S6K1 phosphorylation could be responsible for impaired osteoblast differentiation, the expression of constitutivelyactive S6K1 (CAS6K1, T390E) was enforced in Raptordeficient calvarial osteoblasts. CAS6K1 overexpression was determined by immunoblotting (Figure $5 \mathrm{~h}$ ) and the efficiency of CAS6K1 was confirmed by increased phosphorylation of ribosomal protein S6 (P-S6, S 235/236). Furthermore, the impaired osteoblast differentiation of $\operatorname{Rap}^{O s x}$ calvarial cells was significantly improved by CAS6K1 overexpression as demonstrated by increased ALP activity and mineralized nodule formation (Figure $5 \mathrm{~g}$ ). Similarly, enforced expression of CAS6K1 rescued the decreased expression of Runx2 (Figure 5i), Col1a1 (Figure 5j) and Ocn (Figure 5k) in Raptordeficient calvarial cells. These data demonstrate that the 
impaired S6K1 activity is responsible for the impaired osteoblast differentiation of Raptor-deficient calvarial osteoblasts.
The mTOR-Raptor-S6K1 axis signaling regulates Runx2 expression in osteoblasts. Both $m T O R^{O s x}$ and $R a p^{O s x}$ mice displayed a striking similarity to $R u n \times 2^{+/-}$mice with

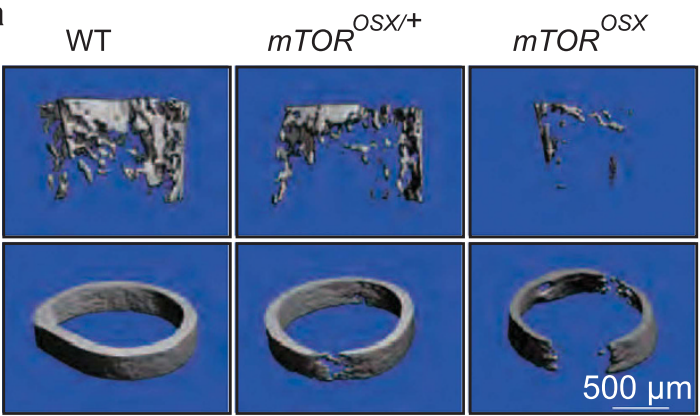

d

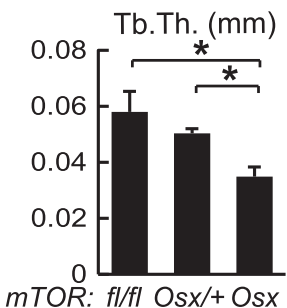

mTOR: fl/fl OsX/+Osx e

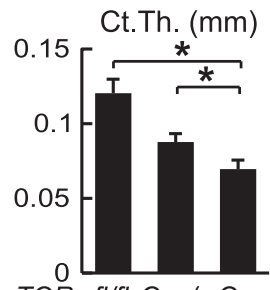

b

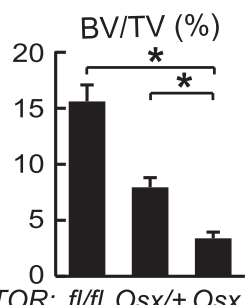

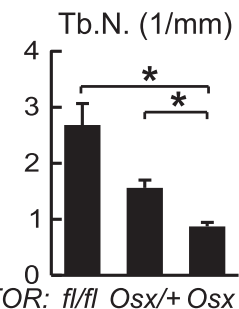
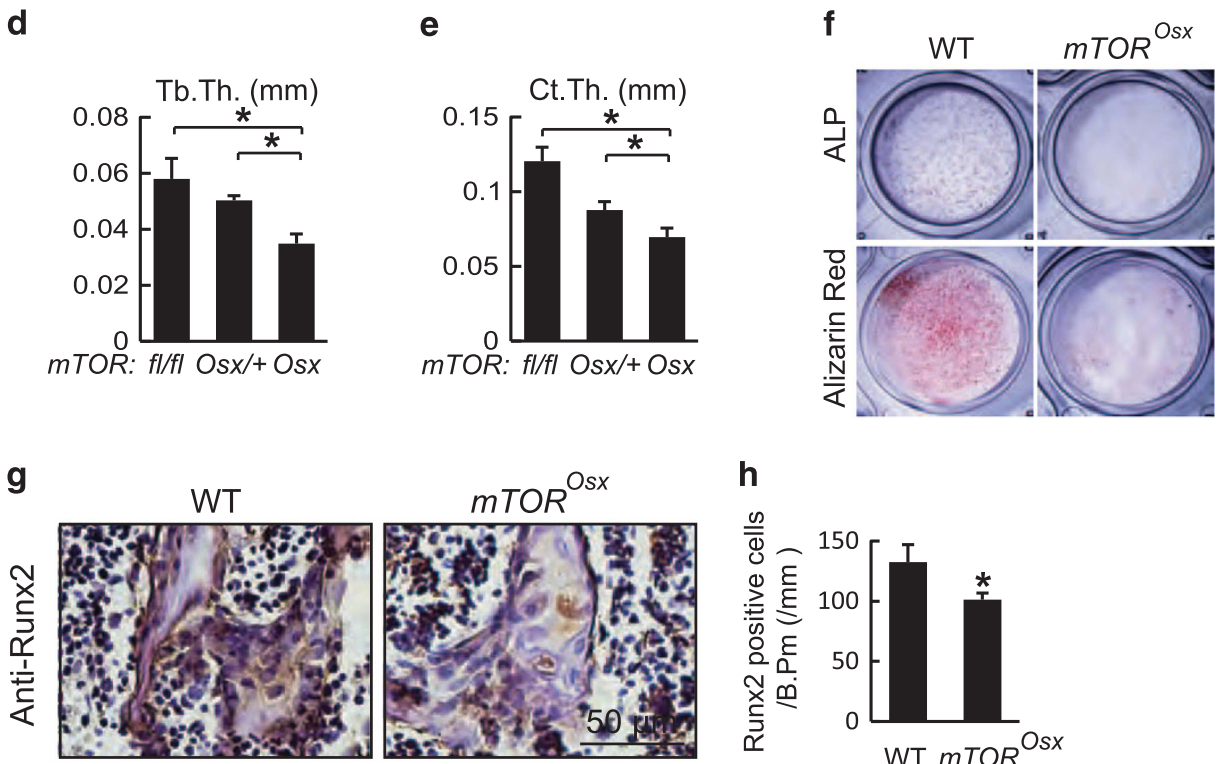

h

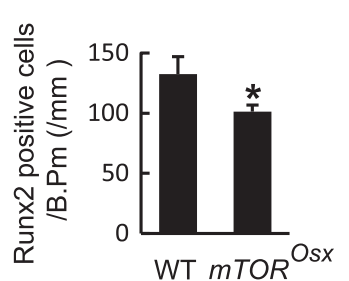

i

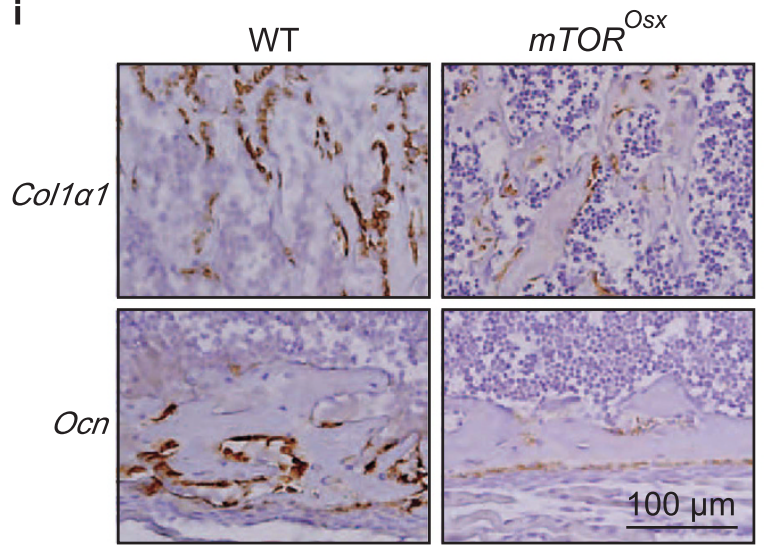

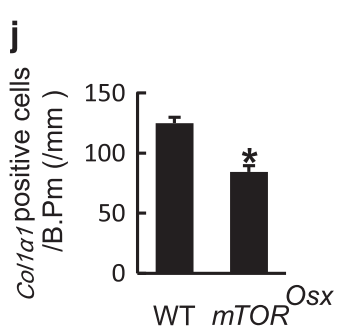

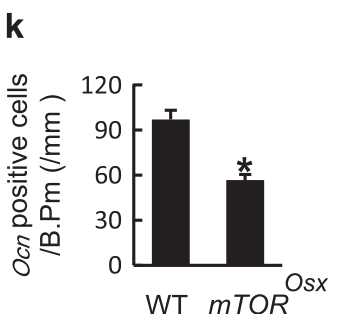

Figure 2 mTOR deficiency in osteoblasts resulted in reduced bone mass with impaired osteoblast differentiation. (a) Micro-CT images of femur from 4-week-old WT, $m T O R^{O s x /+}$ and $m T O R^{O s x}$ littermates. (b-e) Quantitative parameters of Micro-CT. BV/TV: bone volume/total volume, Tb.N.: trabecular number, Tb.Th.: trabecular thickness, Ct.Th.: cortical thickness. Data represent means \pm s.d. ${ }^{*} P<0.05, n=5$. (f) Calvarial osteoblasts were cultured with osteogenic medium from 5-day-old WT and $m T O R R^{O s x}$ littermates. ALP staining and alizarin red staining were performed to analyze osteoblast differentiation on the 7th and 14th day respectively. (g) Immunohistochemistry for Runx2 of distal femur from 7-day-old WT and $m T O R^{\text {Osx }}$ mice. (h) Number of Runx2-positive osteoblasts counted in the distal femur from 7-day-old WT and $m T O R^{O s x}$ mice. Data represent means \pm s.d. ${ }^{*} P<0.05, n=3$. (i) In situ hybridizationfor Col1 $\alpha 1$ and Ocn in femur from 7-day-old WT and $m T O R^{\text {Osx }}$ littermaters. (j) Number of Col1 $\alpha 1$-positive osteoblasts counted in the trabecular bone of distal femur from 7-day-old WT and $m T O R^{O s x}$ littermaters. Data represent means \pm s.d. ${ }^{*} P<0.05, n=3$. (k) Number of $O c n$ positive osteoblasts calculated in the cortical bone of distal femur from 7-day-old WT and $m T O R^{\text {Osx }}$ littermaters. Data represent means \pm s.d. ${ }^{*} P<0.05, n=3$ 
a

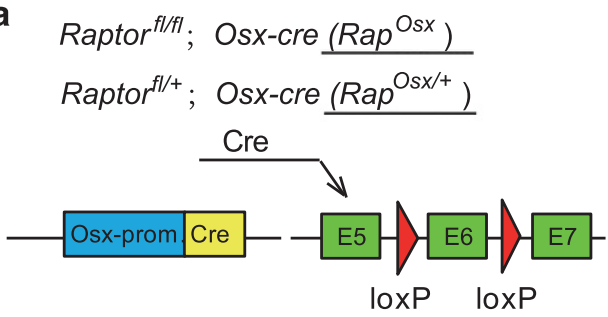

C

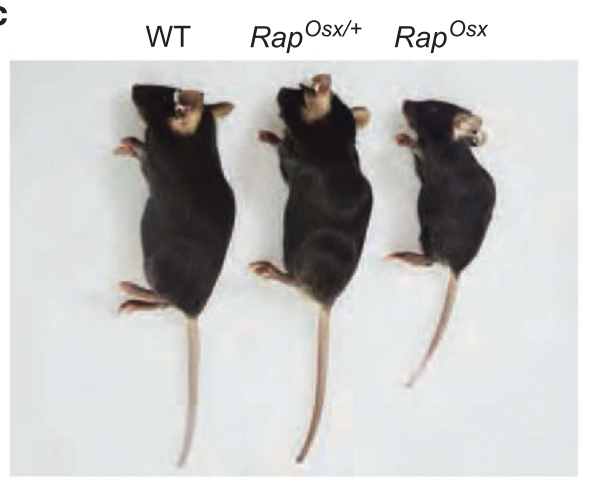

e

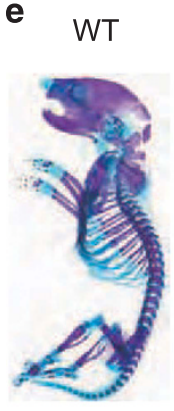

$\operatorname{Rap}^{\mathrm{Osx} /+}$

$\operatorname{Rap}^{\text {Osx }}$
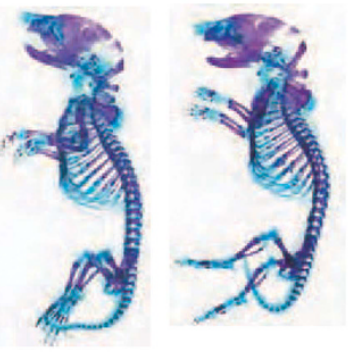

g

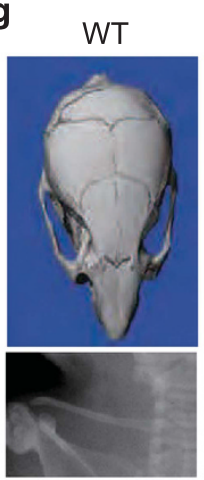

b

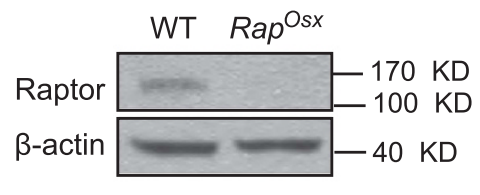

d

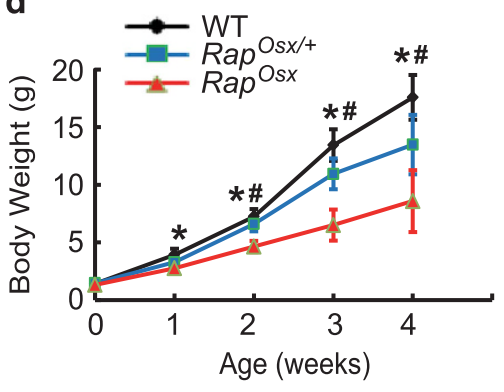

f

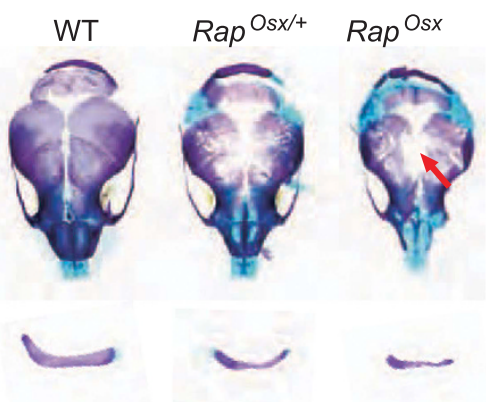

Figure 3 Ablation of Raptor in preosteoblasts resulted in CCD-like phenotype. (a) Illustration of Raptor deletion in Osx-expressing preosteoblasts. (b) Expression of Raptor in parietal bone from WT and Rap ${ }^{O s x}$ newborns. (c) Representative view of 4-week-old WT, Rap ${ }^{O s x /+}$ and Rap ${ }^{O s x}$ mice. (d) Body weight of male WT, Rap ${ }^{O s x /+}$ and Rap ${ }^{O s x}$ littermates measured at different age points $\left(n=5\right.$ for each group, $t$-test). Data represent means \pm s.d. ${ }^{*} P<0.05$ in comparison with WT mice, ${ }^{\#} P<0.05$ when compared with Raposx/+ mice. (e) Representative images of skeleton from WT, Rap ${ }^{O s x++}$ and $R a p^{O s x}$ newborn littermates by alcian blue and alizarin red staining. (f) Clavicular hypoplasia and calvarium hypomineralization of Rap ${ }^{O s x}$ newborns. Arrow indicates calvarial lesions in $m T O R^{O s x}$ mice. (g) Micro-CT of skulls and X-ray of clavicles from 4-week-old WT, Rap ${ }^{O s x /+}$ and Rap ${ }^{\text {Osx }}$ mice. Arrow indicates calvarial lesions and hypoplastic clavicule in $m T O R^{O s x}$ mice

features including CCD phenotype and reduced bone mass, suggesting that mTORC1 may regulate Runx2 expression. This hypothesis was supported by the facts that both $m T O R^{O s x}$ and $R a p^{O s x}$ mice displayed decreased Runx2 protein expression in vivo (Figures $2 \mathrm{~g}$ and $\mathrm{h}$ and Figures $4 \mathrm{~g}$ and $h$ ) and that enforced expression of CAS6K1 increased Runx2 mRNA level (Figure 5i). Likewise, CAS6K1 was able to rescue the decreased Runx2 expression in 
Raptor-deficient osteoblasts at the protein level (Figure 5h). To exclude the possibility that the decreased Runx2 expression is due to the different cell populations, we cultured BMSCs from Raptor ${ }^{f / f l}$ mice and then infected the cells with adenovirus expressing GFP and CRE recombinase. As shown in Figure 6a, CRE adenovirus led to reduced expression of Raptor accompanied by decreased levels of Runx2 protein. Thus, the effect of Raptor on regulating Runx2 expression cannot be attributed to differences in cell population. Consistent with the lower Runx2 protein level, CRE adenovirus caused impaired osteoblast differentiation (Figure $6 \mathrm{~b}$ ). The change of Runx2 protein level was reflected in a dramatic decrease of Runx2 mRNA level by CRE adenovirus (Figure 6c), which was accompanied by a decrease of Runx2 downstream osteogenic genes such as Col1a1(Figure 5d) and Ocn (Figure 5e). These data suggest that mTORC1 could regulate Runx2 expression at the transcript level. To further determine whether the impaired osteoblast differentiation induced by inactivation of mTORC1 was due to the reduction of Runx2 expression, we analyzed the effects of enforced Runx2 expression on Raptor-deficient osteoblasts. Calvarial osteoblastic cells from Rap ${ }^{O s x}$ mice were infected with lentivirus expressing Runx2 (Lenti-Runx2) or GFP (Lenti-GFP). Western blot assay confirmed Runx2 overexpression in Rap Osx osteoblasts (Figure 6f). The decreased osteoblast differentiation in $\operatorname{Rap}^{O s x}$ calvarial cells was rescued by Runx2 overexpression as determined by ALP activity and mineralized nodule formation (Figure $6 \mathrm{~g}$ ). Consistent with this, the decreased expression of Runx2 downstream genes including Col1a 1and Ocn was rescued by Runx2 overexpression (Figures $6 \mathrm{~h}$ and i). These results indicated that mTOR/Raptor-S6K1 signaling promoted skeletal development and osteoblast differentiation through regulation of Runx2 expression.

S6K1 regulates Runx2 expression via its enhancer. Next, we intended to investigate the mechanisms by which mTORC1 regulates Runx2 expression. First, we examined the effects of CAS6K1 on activity of the Runx 2 promoter by an in vitro luciferase assay. As shown in Supplementary Figure 3A, CAS6K1 had no effects on activation of the Runx2 promoter. Kawane et al. demonstrated that the enhancer of Runx2 plays an important role in directing Runx2 expression in osteoblasts. ${ }^{23}$ Consequently, we next examined the effects of CAS6K1 on the Runx2 enhancer. As shown in Figure 7a, the activity of the luciferase reporter driven by the $3 x 89 \mathrm{bp}$ Runx2 core enhancer (Runx2 enhancer) was promoted by CAS6K1 expression. Furthermore, we found Runx2 enhancer activity decreased in $\operatorname{Rap}^{\text {Osx }}$ calvarial cells compared with WT group (Figure 7b), which confirmed mTORC1-S6K1may regulate Runx2 expression through its enhancer. The Runx2 core enhancer is bound by Distal-less homeobox 5 (DLX5) and MEF2C, ${ }^{23}$ so we examined the effects of CAS6K1 on DLX5- and MEF2C-induced Runx2 enhancer activity. As shown in Figure 7c, CAS6K1 could increase DLX5-induced Runx2 enhancer activity, but not MEF2C (Supplementary Figure 3B). However, we were unable to detect any interaction between S6K1 and DLX5 by co-immunoprecipitation (colP) experiments (Supplementary Figure $1 \mathrm{C}$ ). This is consistent with the fact that DLX5 does not contain the S6K1 phosphorylation motif RxRxxS/T (where $x$ could be any amino acid). Thus, S6K1 may regulate Runx2 enhancer activity by phosphorylating proteins other than DLX5. It has been reported that S6K1 promotes estrogen receptor a (ERa) activity by phosphorylating it on $\mathrm{S} 167$ in cancer cells ${ }^{9,24}$ and $\mathrm{ER} a$ can regulate Runx2 expression in osteoblast progenitors. ${ }^{25-27}$ We hypothesized that S6K1 may regulate Runx2 enhancer activity by phosphorylating ERa. First, we confirmed the protein interaction between S6K1 and ERa (Figure 7d). Next, we found that ERa could increase the activity of Runx2 enhancer, while co-transfection of CAS6K1 was able to promote this effect (Figure 7e).We then examined whether mutation of the S6K1 phosphorylation motif renders ERa refractory to activation by S6K1. The S6K1 phosphorylation site of ERa was mutated by converting Ser ${ }^{171}$ into alanine. ${ }^{28}$ We found that when compared with WT ER $a$, ER $a$ S171A was resistant to CAS6K1 effects on the activity of Runx2 enhancer (Figure 7f). These data indicate that S6K1 regulates Runx2 enhancer activity by phosphorylation of ERa. To further test this hypothesis, we analyzed the phosphorylation of ERa (P-ERa, S167) in 7-day-old $m$ TOR ${ }^{\text {Osx }}$ and $\operatorname{Rap}^{O s x}$ mice by immunohistochemistry. As shown in Supplementary Figures 3D,E and Figures $7 \mathrm{~g}$ and $\mathrm{h}, \mathrm{P}-\mathrm{ER} a-$ positive cells decreased in both $m T O R^{O s x}$ and Rap ${ }^{O s x}$ mice compared to WT mice, which confirmed that S6K1 regulates Runx2 enhancer activity by phosphorylation of ERa.

Interestingly, we found that DLX5 and ERa could synergistically increase the activity of Runx2 enhancer and that CAS6K1 could further promote these effects (Figure 7i). As the 89 bp Runx2 core enhancer lacks an ERa binding motif, we hypothesized that ERa might augment Runx2 enhancer activity through interaction with DLX5. Indeed ER $a$ and DLX5 could interact when both were ectopically expressed in 293T cells (Figure 7j). Next, we confirmed this protein interaction between ER $a$ and DLX5 in calvarial osteoblasts (Figure 7k). To further analyze whether the interaction of ERa and DLX5 was dependent on phosphorylation of ERa, WTand phosphorylation site mutant ERa (ERa-WT, ERa-S171E, ERa-S171A) were co-transfected with DLX5 in 293T cells. As shown in Figure 7I, DLX5 could interact with all three ERa protein, which suggested that the interaction of ER $a$ and DLX5 was independent of ERa phosphorylation and excluded the possibility that $\mathrm{mTORC} 1$ regulate Runx 2 enhancer activity by affecting the interaction of ERa and DLX5. Next we analyze the binding of ERa and DIx5 to Runx2 enhancer by CHIPqPCR in Rap ${ }^{\text {Osx }}$ calvarial osteoblasts. As shown in Figures $7 \mathrm{~m}$ and $\mathrm{n}$, the binding of ERa and DIx5 to Runx2 enhancer decreased in Rap ${ }^{\text {sx }}$ calvarial osteoblasts in comparison to the WT groups, which indicated that mTORC1-S6K1 signaling can promote the binding of the transcriptional factors ER $a$ and DIx5 to Runx2 enhancer. These data support the hypothesis that the mTOR-Raptor-S6K1 axis can regulate Runx2 expression via its enhancer, probably by phosphorylating ER $a$ which interacts with DLX5 and augments Runx2 enhancer activity.

mTORC1 interacts genetically with Runx2 in vivo. Based on the in vitro results above, we next questioned whether mTORC1 is an upstream regulator of Runx2 in vivo. We postulated that if $\mathrm{mTORC} 1$ indeed regulates expression of 


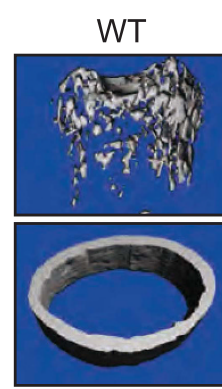

d

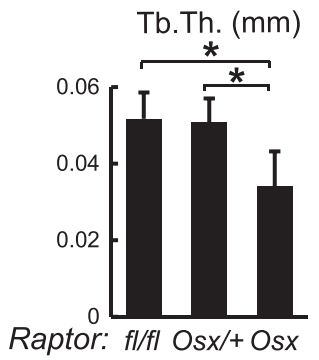

g

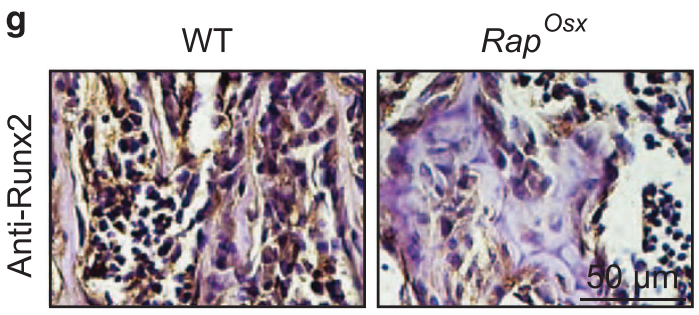

i

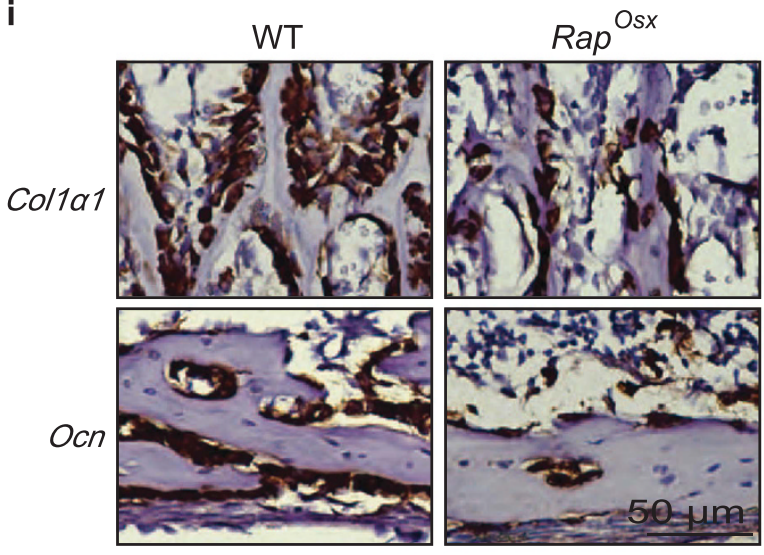

I

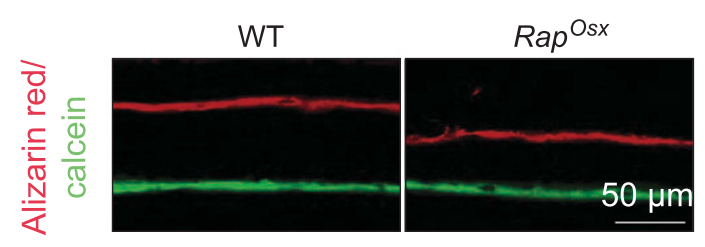

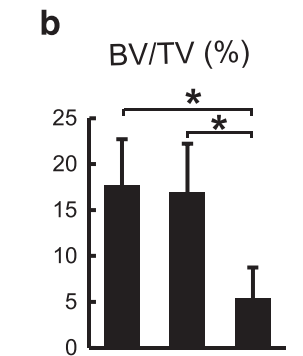

Raptor: $f / / f \mid \mathrm{Os} x /+\mathrm{Osx}$
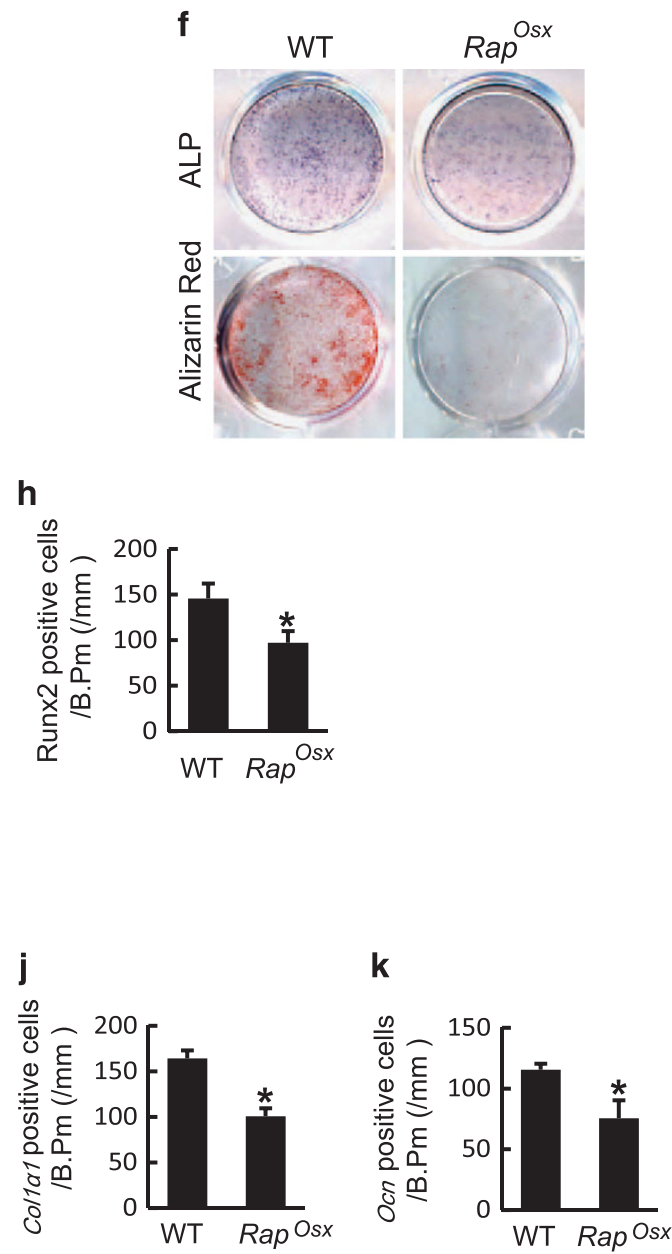

m

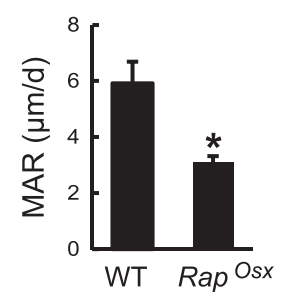


Runx2, then reduced activity of mTORC1 in parallel with Runx2 haploinsufficiency in vivo should aggravate the bone

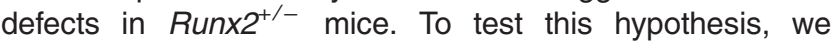

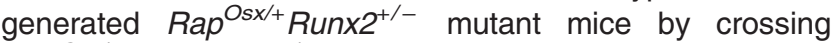
Rap ${ }^{O s x /+}$ and $R u n x 2^{+/-}$mice and analyzed skeletal preparations from newborn WT, Rap ${ }^{O s x /+}, R_{u n x 2^{+/-}}$and $\mathrm{Rap}^{\mathrm{Osx} /+} \mathrm{Runx}^{+/-}$mice. As shown in Figure $8 \mathrm{a}$, the body

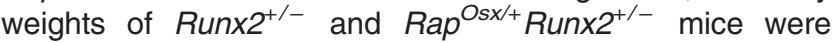
slightly lower than those of WT and $\operatorname{Rap}^{\mathrm{Osx/+}}$ mice and the skeletons of Runx $2^{+/-}$and Rap ${ }^{O s x /+} R u n x 2^{+/-}$mice were consistently smaller (Figure 8b). Run $x 2^{+/-}$mice displayed the previously reported CCD-like phenotype. Heterozygous deletion of Raptor resulted in minor skeletal defects in both the clavicle and calvarium (Figures $8 \mathrm{c}-\mathrm{e}$ ). However, Rap ${ }^{\mathrm{sx} /+}$ Run $x 2^{+/-}$mice showed far more severe bone defects than Run $\times 2^{+/-}$mice, including a larger hypoplastic area of the cranial bones (Figures $8 \mathrm{c}$ and $\mathrm{d}$ ) and barely visible clavicles (Figures $8 \mathrm{c}$ and e). Taken together, these results suggest a genetic relationship between $\mathrm{mTORC} 1$ and Runx2, and provide in vivo evidence that $\operatorname{mTORC1}$ can regulate osteoblast function through Runx2.

\section{Discussion}

In the present study, we revealed the critical role of mTOR/ Raptor signaling in osteoblasts and bone development through conditional knockout of mTOR and Raptor, respectively. Deletion of $m T O R$ in preosteoblasts induced marked skeletal defects, including dwarfism with short limbs, impaired ossification of the cranial bones, hypoplasia of the clavicles and reduced bone mass, which support the conclusion that mTOR is essential for both endochondral and intramembranous ossification. Further, preosteoblast-specific loss of Raptor reproduced almost all skeletal phenotypes of $m T O R^{O s x}$ mice, suggesting that mTOR functions mainly though mTORC1 in osteoblasts and osteogenesis. On the other hand, other researchers have found that continuous mTORC1 activation led to bone defects. ${ }^{18,19}$ These results suggested that mTOR signaling is regulated precisely during bone development, and either upregulation or downregulation of mTORC1 signaling may result in bone diseases. Although the precise balance point of $\mathrm{mTORC} 1$ signaling in osteoblasts will need to be investigated in further studies, the possible side effects on bone of changes in mTORC1 signaling caused by any agent which has been or is intended to be used as a treatment target in a range of diseases ${ }^{11}$ should be taken into account.

MSCs can differentiate into multiple lineages including the osteoblast, chondrocyte and adipocyte lineages. While it has been demonstrated that mTOR signaling is essential for both chondrocyte $^{20,29}$ and adipocyte ${ }^{30}$ differentiation, there is limited and controversial information available regarding the independent role of mTOR in osteoblast and osteogenesis. ${ }^{12}$ In this study, we revealed several lines of evidence showing that inactivation of mTORC1 inhibited osteoblast differentiation and bone formation. First, osteoblast-specific deletion of either mTOR or Raptor led to decreased expression of osteoblastic markers in vivo. Second. BFR was reduced in Rap ${ }^{O s x}$ mice. Third, osteoblast differentiation of Rap Osx parietal cells was impaired. Taken together, we believe that physiological $\mathrm{mTORC} 1$ signaling is essential for osteoblast differentiation and bone formation.

There is limited information available about the mechanisms by which $\mathrm{mTORC} 1$ promotes osteoblast differentiation. It has been demonstrated that S6K1can positively regulate the differentiation of both chondrocytes ${ }^{29}$ and adipocytes. ${ }^{10}$ In our current study, we provided evidence indicating that S6K1 is the major downstream regulator of mTORC1 in osteoblasts and that the mTOR/Raptor-S6K1 axis could promote osteoblast differentiation and osteogenesis.

Mutations of Runx2 result in CCD in humans and $R u n \times 2^{+/-}$ mice exhibited CCD-like phenotypes except for supernumerary teeth. ${ }^{4-6}$ However, some CCD patients do not carry Runx2 mutations, and a decrease to $70 \%$ of wild-type Runx 2 levels can result in CCD syndrome in mice. ${ }^{31}$ These results indicate that $C C D$ may result from other mechanisms that regulate Runx2 expression. Here, we provided evidence that mTOR/ Raptor-S6K1 signaling promotes Runx2 expression. Preosteoblast-specific deletion of either mTOR or Raptor results in bone defects, resembling the CCD phenotype and decreased Runx2 expression in vivo. Moreover, Raptordeficient BMSCs and parietal cells displayed reduced Runx2 expression, and the impaired osteoblast differentiation in Rap ${ }^{\text {Osx }}$ parietal cells can be rescued by Runx2 overexpression. The reduced Runx 2 expression in Rap ${ }^{O s x}$ parietal cells can be rescued by enforced CAS6K1 expression. Molecular experiments demonstrated that CAS6K1 can augment the activity of Runx2 enhancer, but not the Runx2 promoter. Finally, heterozygous Raptor in osteoblasts aggravates skeletal phenotypes of $R u n \times 2^{+/-}$mice, supporting a genetic link between mTORC1 signaling and Runx2. Taken together, these data suggest that physiological mTORC1 signaling is essential for Runx2 expression. Meanwhile, hyperactivation of mTORC1 in osteoblasts-induced bone defects were also related to decreased Runx2 expression. ${ }^{18,19}$

Our study clarifies the critical role of mTOR/Raptor signaling in osteoblasts and bone development and demonstrates that mTOR-Raptor-S6K1 can regulate Runx2 expression, providing new sights into skeletal dysplasias such as CCD.

Figure 4 Raptor deficiency in osteoblasts displayed reduced bone mass with impaired osteoblast differentiation. (a) Micro-CT images of femur from 4-week-old WT, Rap Osx/+ and Rap ${ }^{\text {Ssx }}$ littermates. (b-e) Quantitative parameters of Micro-CT. Data represent means \pm s.d. ${ }^{*} P<0.05, n=5$. (f) ALP staining and alizarin red staining of WT and Rap ${ }^{O s x}$ calvarial cells induced by osteogenic medium. Data represent means \pm s.d. ${ }^{*} P<0.05, n=3$. (g) Immunohistochemistry for Runx2 of distal femur from 7-day-old WT and Rap ${ }^{O s x}$ mice. (h) Number of Runx2-positive osteoblasts counted in the trabecular bone of distal femur from WTand Rap ${ }^{\text {Osx }}$ mice. Data represent means \pm s.d. ${ }^{*} P<0.05, n=3$. (i) In situ hybridizationfor Col1 1 and Ocn in femur from 7-day-old WTand Rap ${ }^{\text {sxx }}$ littermaters. (j) Number of Col1 1 1-positive osteoblasts counted in the trabecular bone of distal femur of 7-day-old WTand Rap ${ }^{O s x}$ littermaters. Data represent means \pm s.d. ${ }^{*} P<0.05, n=3$. (k) Number of Ocn-positive osteoblasts counted along the cortical bone of distal femur from 7-day-old WT and Rap ${ }^{O s x}$ littermaters. Data represent means \pm s.d. ${ }^{*} P<0.05, n=3$. (I) Representative images of alizarin red and calcein double labeling of femur from WTand Rap $^{\text {Osx }}$ littermates. $(\mathrm{m}, \mathrm{n})$ Quantitative parameters mineral apposition rate (MAR) and bone formation rate (BFR) in WT and RapOsx mice. Data represent means $\pm \mathrm{s} . \mathrm{d}$. ${ }^{*} P<0.05$, $n=3$ 


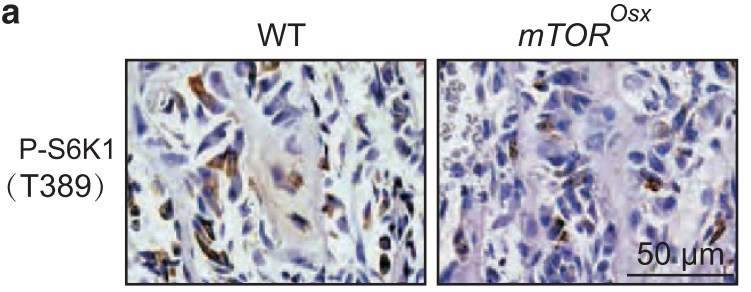

d

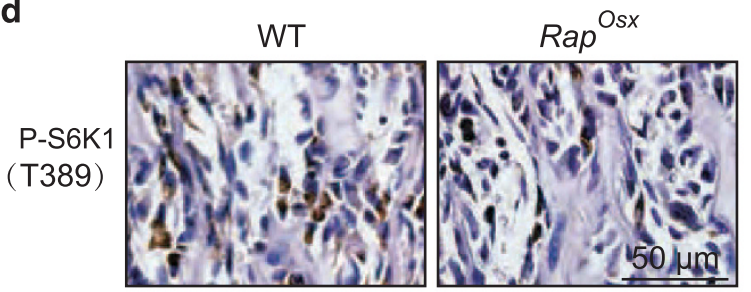

g

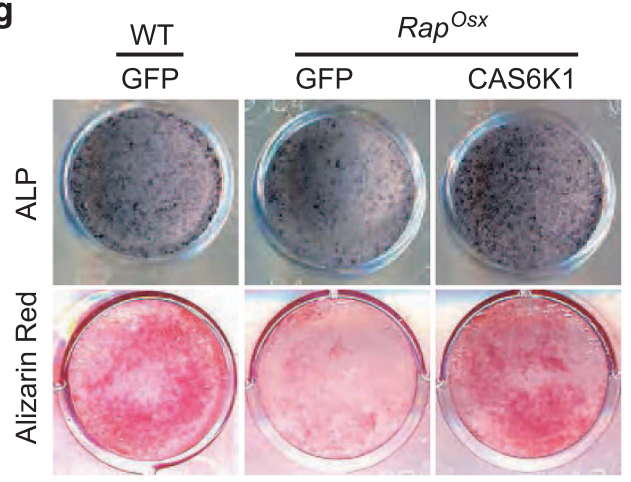

i
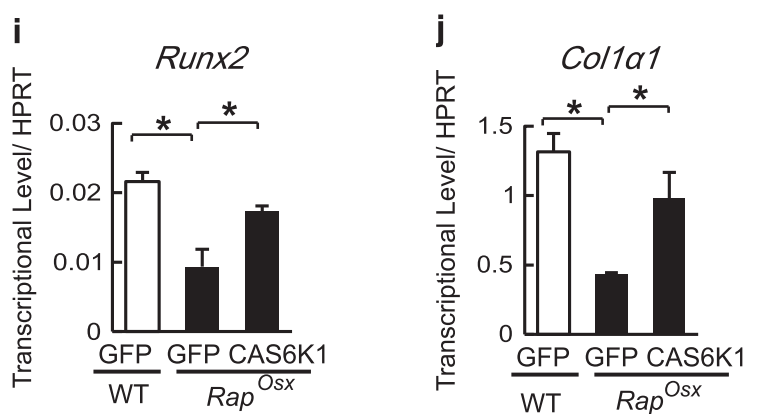

C

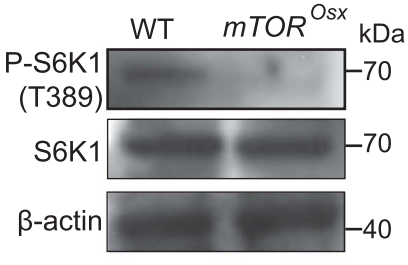

f

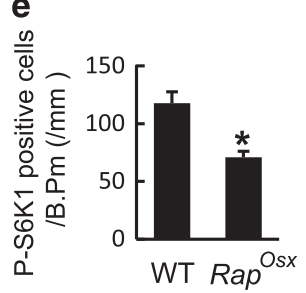

h
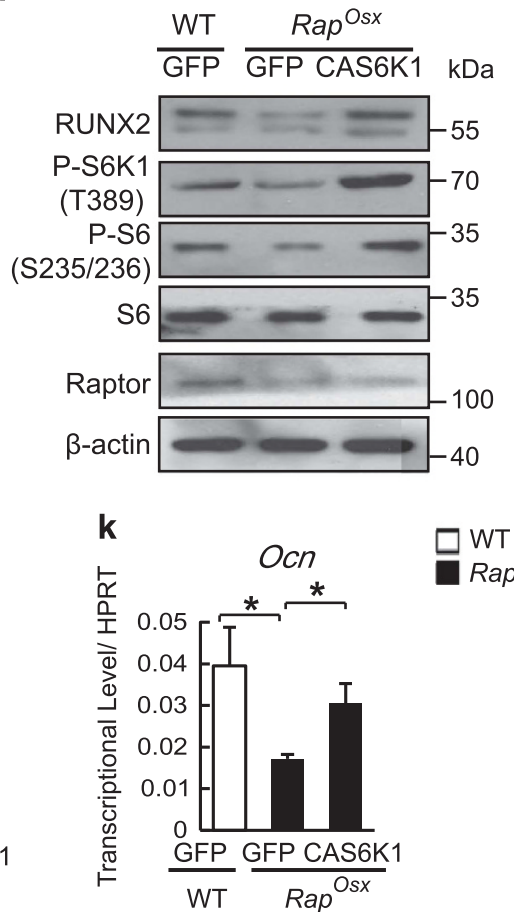

Figure 5 S6K1 is the downstream factor of mTOR/Raptor in osteoblasts. Immunohistochemistry for P-S6K1(T389) in femur from 7-day-old WTand $m T O R^{O s x}$ littermaters. (a) Number of P-S6K1-positive osteoblast counted in the distal femur of WTand $m T O R^{O s x}$ mice. Data represent means \pm s.d. ${ }^{*} P<0.05, n=3$. (b) Western blot assay for cell lysates from parietal bone of WTand TOR ${ }^{\text {ssx }}$ newborn mice. IHC for phospho-S6K1 in femur from P7 WTand Rap ${ }^{\text {Sx }}$ littermaters. (c) Immunohistochemistry for P-S6K1(T389) in femur of 7-day-old WT and Rap ${ }^{O s x}$ littermaters. (d) Number of P-S6K1-positive osteoblast counted in the distal femur of WT and Rap ${ }^{O s x}$ mice. Data represent means \pm s.d. ${ }^{*} P<0.05$, $n=3$. (e) Western blot assay for cell lysates from parietal bone of WT and Rap ${ }^{\text {ssx }}$ newborns. (f) ALP staining and alizarin red staining of WT and Rap $p^{\text {ssx }}$ calvarial cells that were infected with lentivirus expressing CAS6K1 or GFP and induced by osteogenic medium. (g) Western blot assay for cell lysates from WT and Rap ${ }^{\text {Osx }}$ calvarial cells that were infected with lentivirus expressing CAS6K1 or GFP followed by osteogenic medium. (i-k) The relative mRNA levels of Runx2, Col1 $\alpha 1$ and Ocn were quantified by qPCR. Data represent means \pm s.d. ${ }^{*} P<0.05, n=3$

\begin{abstract}
Materials and Methods
Mice. $m T O R^{f / f t l}$ mice bearing loxP sites flanking exons $1-5$ of the $m T O R$ gene (Stock No: 011009) and Rap $p^{t / f t}$ mice bearing loxP sites flanking exons 6 of the Raptor gene (Stock No: 013188) were purchased from the Jackson Laboratory (Bar Harbor, Maine, USA). TTOR $^{f / f t}$ mice were crossed with Osx-cre mice (Osx1-GFP::Cre mice were purchased from the Jackson Laboratory (Stock No: 006361)) to generate $m T O R^{t / f t} ;$ Osx-cre $\left(m T O R^{O s x}\right)$ and $m T O R^{f / /} ;$ Osx-cre $\left(m T O R^{O s x /+}\right)$ mice. Rap ${ }^{f / f l}$ were crossed with Osx-cre mice
\end{abstract}

to generate Raptor ${ }^{f / / f t} ;$ Osx-cre $\left(\right.$ Rap $\left.{ }^{\text {Osx }}\right)$ and Raptorl ${ }^{f /+} ;$ Osx-cre (Rap ${ }^{\text {Osx++ }}$ ) mice. Runx $2^{+/-}$mice were kindly provided by Professor Gerard Karsenty's laboratory. All mice were C57BL/6 background. All mice were bred and maintained under specific pathogen-free conditions in the institutional animal facility of the Shanghai Institute of Biochemistry and Cell Biology, Chinese Academy of Sciences. All experiments were performed with the protocol approved by the Animal Care and Use Committee of the Shanghai Institute of Biochemistry and Cell Biology. 
a

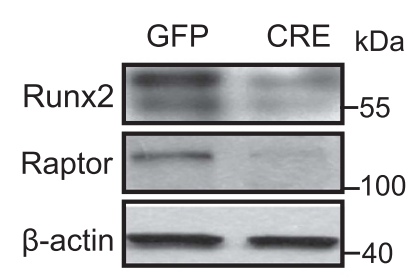

b
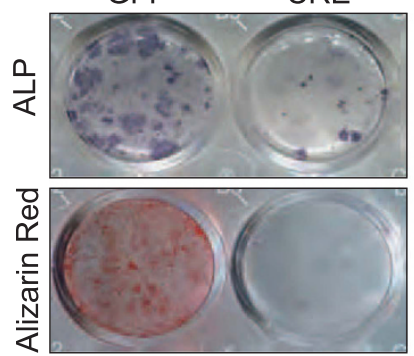

C

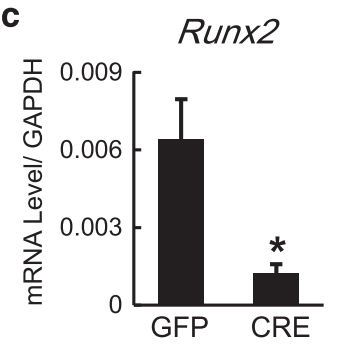

d

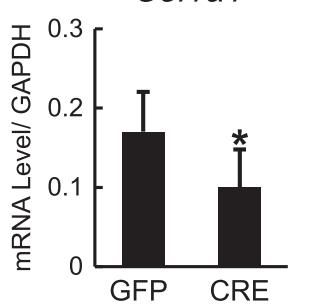

e

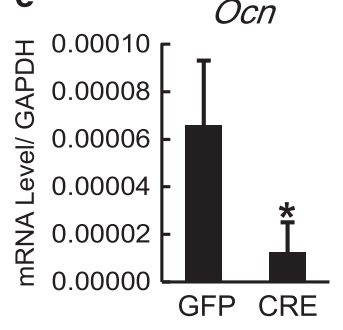

g
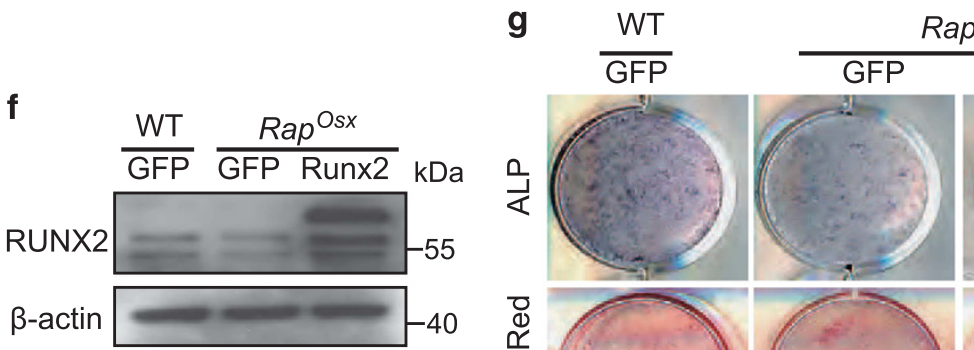

Rap Osx
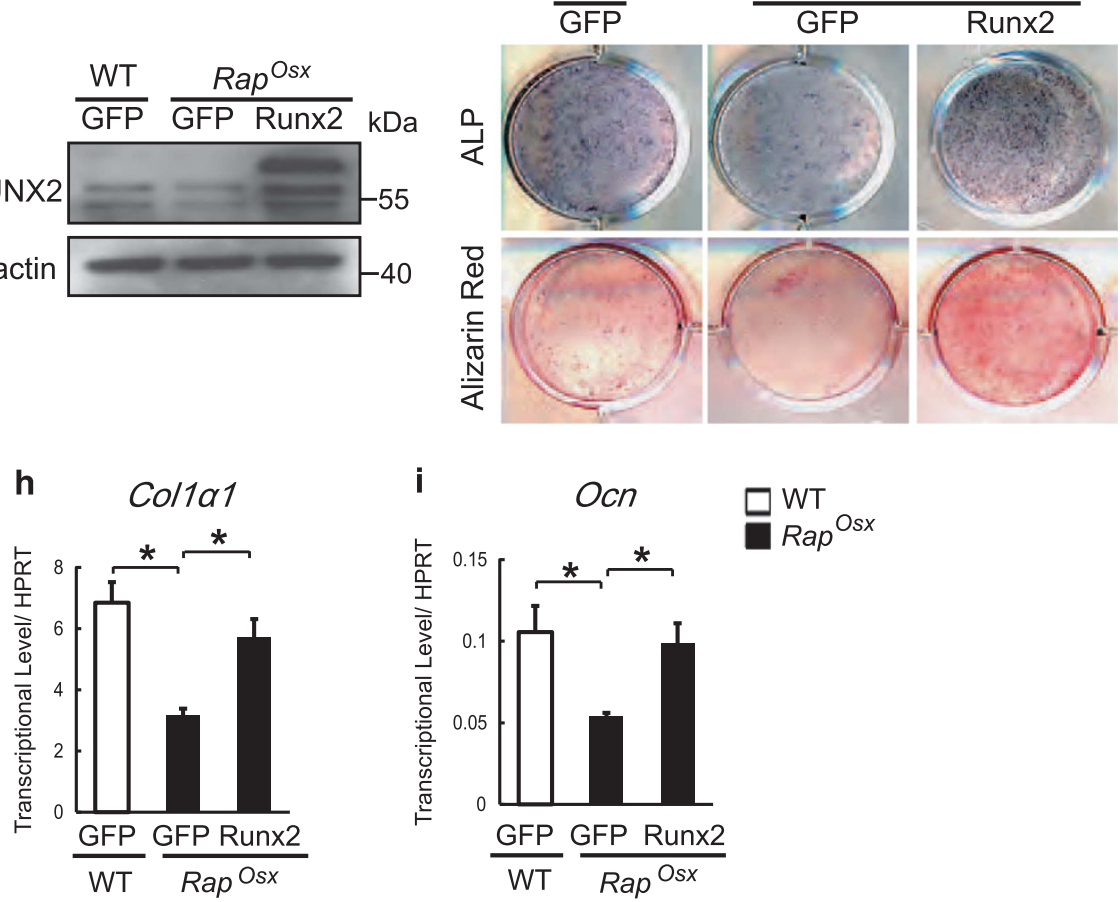

Figure 6 mTOR/Raptor-S6K1 signaling regulates Runx2 expression in osteoblast. (a) Western blot assay for cell lysates from Raptor ${ }^{f / f l l}$ BMSCs that were infected with adenovirus expressing GFP and CRE recombinase. (b) ALP staining and alizarin red staining of BMSCs induced by osteogenic medium. (c-e) The relative mRNA levels of Runx2, Col1 $\alpha 1$ and Ocn were quantified by qPCR. Data represent means \pm s.d. ${ }^{*} P<0.05, n=3$. (f) Western blot assay for cell lysates from WT and Rap ${ }^{\text {osx }}$ calvarial cells infected with lentivirus expressing Runx2 or GFP. (g) ALP staining and alizarin red staining of WT and Rap ${ }^{O s x}$ calvarial cells that were infected with lentivirus expressing Runx2 or GFP and induced by osteogenic medium. (h-i) The relative mRNA levels of Col1 11 and Ocn were quantified by qPCR. Data represent means \pm s.d. ${ }^{*} P<0.05, n=3$

Skeletal whole mount staining. Skeletal whole mount staining with Alcian blue and Alizarin red was performed as described previously. ${ }^{32}$ Mice were killed with $\mathrm{CO}_{2}$, and all skin was carefully removed. Specimens were dehydrated in $95 \%$ alcohol for $24 \mathrm{~h}$, followed by cartilage staining in Alcian blue solution for $42 \mathrm{~h}$ at $37{ }^{\circ} \mathrm{C}$. After staining, specimens were washed twice in $95 \%$ alcohol for $2 \mathrm{~h}$, cleared in $1 \% \mathrm{KOH}$ for $5 \mathrm{~h}$ and stained in Alizarin red solution for $1 \mathrm{~h}$. They were then cleared through 20,50 , and $80 \%$ glycerine in $1 \% \mathrm{KOH}$, then stored in glycerine.
X-ray and micro-CT analysis. Four-week-old mice were anesthetized with chloral hydrate and subjected to X-ray scanning at $30 \mathrm{KV}$ (Faxitron X-ray, Tucson, AZ, USA). Skulls of 4-week-old and 6-month-old WT and Rap ${ }^{\text {sx }}$ mice were used for micro-CT analysis ( $\mu$ CT80, SCANCO Medical AG, Bassersdorf, Switzerland) with a $10-\mu \mathrm{m}$ voxel size. The femur of 4-week-old $m T O R^{O s x}, m_{T O R}{ }^{O s x++}, R^{O s}$, Rap ${ }^{O s x+x}$ mice, 6-month-old Rap ${ }^{O s x}$ mice and corresponding WT littermates were collected for micro-CT scanning with a $10-\mu \mathrm{m}$ voxel size. One hundred slices (total 
1 voxel size. One hundred slices (total 1lected for micro-CT scanning witrabecular microarchitecture parameters including bone volume fraction (BV/TV), trabecular thickness (Tb.Th.) and trabecular number (Tb.N.) following the introductions of the manufacturer. ${ }^{33,34}$ Fifty slices from the middle of the femur were used to analyze cortical thickness (Ct.Th.). ${ }^{35}$
Histological analysis. Femurs from 7-day-old and 4-week-old mice were fixed in $4 \%$ paraformaldehyde for $48 \mathrm{~h}$ followed by decalcification in 10\% EDTA for 2-4 weeks. Specimens were embedded in paraffin then stained with hematoxylineosin and TRAP (Sigma, St. Louis, MO, USA) according to previously described methods. ${ }^{36,37}$ a

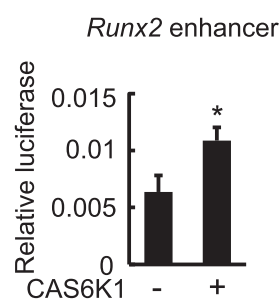

d

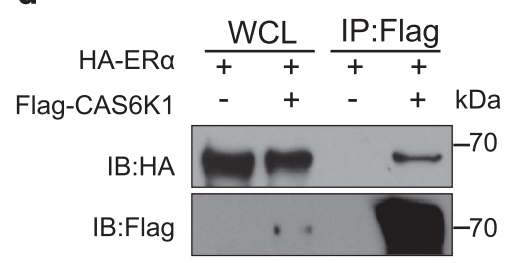

b

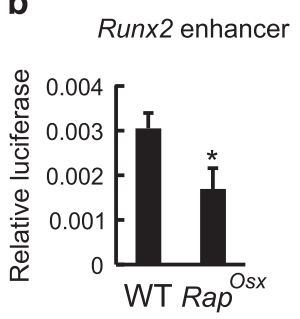

e

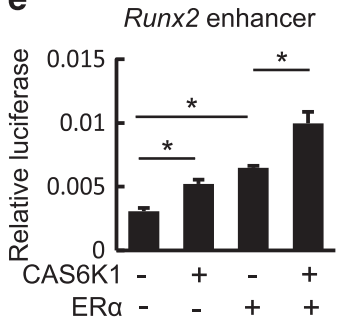

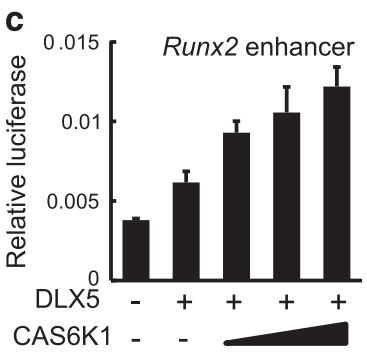

CAS6K1 -

f

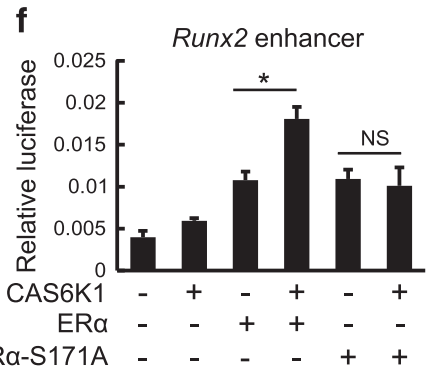

g

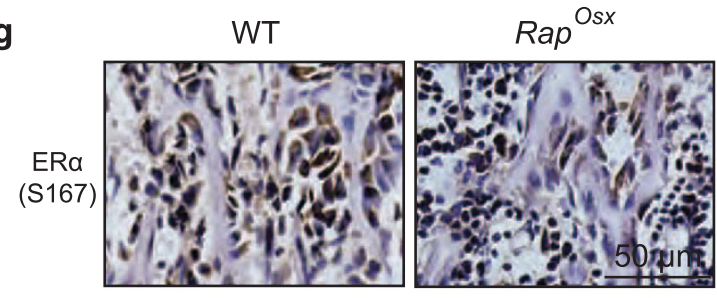

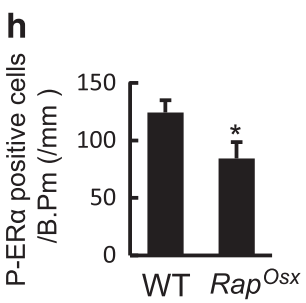

k

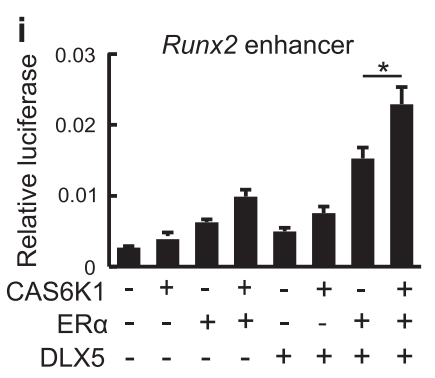

I

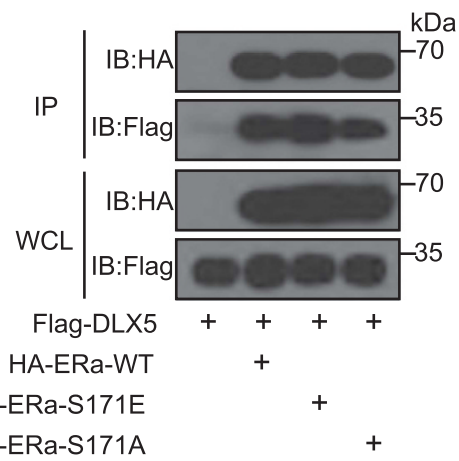

j

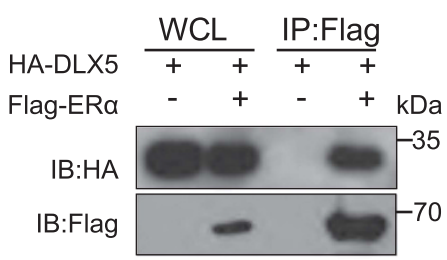

m

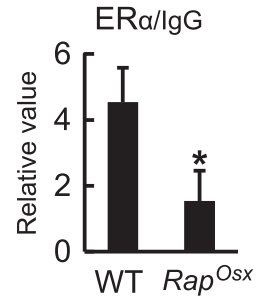

IB: DLX5

$W C L \frac{\mathrm{IP}}{\lg \quad \mathrm{ER} \alpha \mathrm{kDa}}$

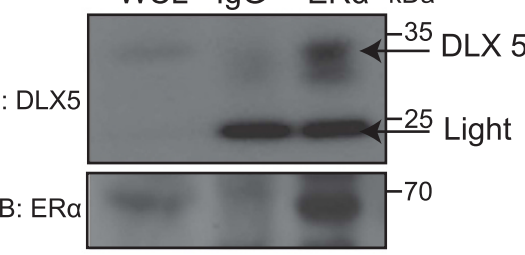

n

DLX5/lgG

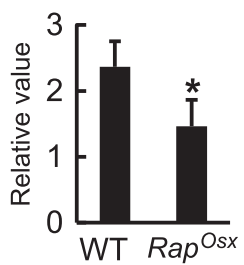


a

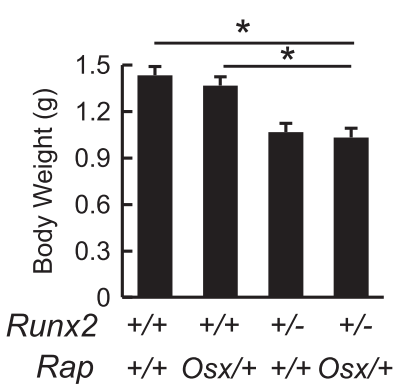

b

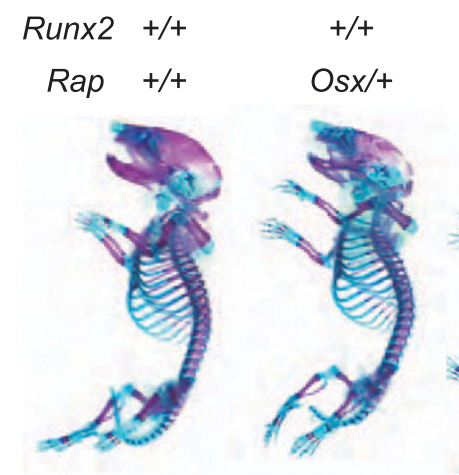

+/-

$+/+$

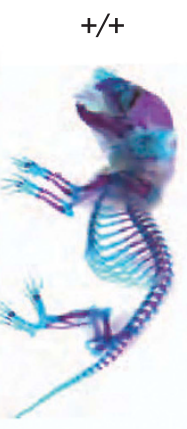

+/-

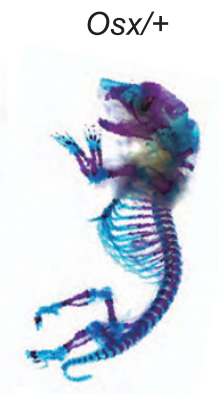

d

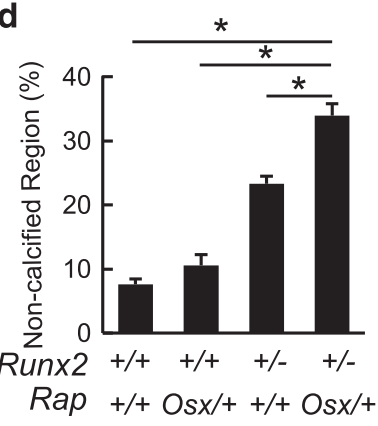

e

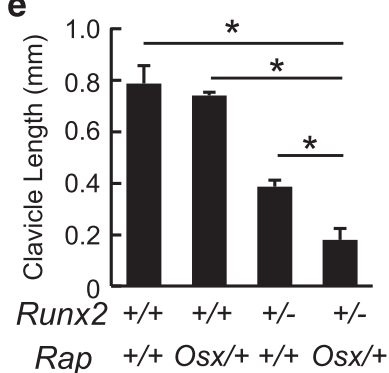

Figure 8 mTORC1 genetically interact with Runx2. (a) Body weight analysis of neonatal WT, Rap ${ }^{O s x /+}$, Runx $2^{+/-}$, and Rap ${ }^{O s x /+}$ Runx $^{+/-}$mice. (b) Alcian blue and alizarin

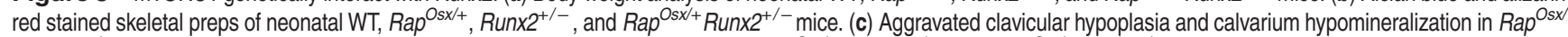
${ }^{+}$Runx $2^{+/-}$mice. (d) Relative non-calcified area of calvarial bones of neonatal WT, Rap ${ }^{O s x /+}$, Runx $2^{+/-}$, and Rap ${ }^{O s x /+}$ Runx $^{+/-}$mice. (e) Length of clavicles of neonatal WT, Rap $^{O s x /+}$, Runx $2^{+/-}$, and Rap ${ }^{O s x /+}$ Runx $2^{+/-}$mice. Data represent means \pm s.d. ${ }^{*} P<0.05, n=3$

Figure 7 S6K1 regulates Runx2 expression via its enhancer. (a) Effects of CAS6K1 on Runx2 enhancer activity. 3X 89 bp Runx2 enhancer corn sequence-driven luciferase reporter and pRL-TK were co-transfected with or without CAS6K1 in C3H10T1/2 cells. Data represent means \pm s.d. ${ }^{*} P<0.05, n=3$. (b) Effects of Raptor deficiency on Runx2 enhancer activity. 3X 89 bp Runx2 enhancer corn sequence-driven luciferase reporter and pRL-TK were co-transfected in WT and Rap ${ }^{\text {Osx }}$ calvarial cells. Data represent means \pm s.d. ${ }^{*} P<0.05, n=3$. (c) CAS6K1 promoted DLX5-induced Runx2 enhancer activity. $3 X 89$ bp Runx2 enhancer corn sequence-driven luciferase reporter and pRL-TK were co-transfected with DLX5 and different concentration of CAS6K1 in C3H10T1/2 cells. Data represent means \pm s.d. ${ }^{*} P<0.05, n=3$. (d) Co-immunoprecipitation analysis of S6K1 and ER $\alpha$. Flag-CAS6K1 and HA-ER $\alpha$ expression constructs were co-transfected in 293Tcells for $48 \mathrm{~h}$ and co-immunoprecipitation analysis was performed. (e) CAS6K1 promoted ER $\alpha$-induced Runx2 enhancer activity. 3X 89 bp Runx2 enhancer corn sequence-driven luciferase reporter and pRL-TK were co-transfected with ER $\alpha$ and CAS6K1 in C3H10T1/2 cells. Data represent means \pm s.d. ${ }^{*} P<0.05, n=3$. (f) Effects of mutated ER $\alpha$ of S6K1 phosphorylation motif on Runx2 enhancer activity. Luciferase assay was performed by co-translating CAS6K1 plasmid with either ER $\alpha$ or ER $\alpha \mathrm{S} 171 \mathrm{~A}$, the later one could not be phosphorylated by S6K1. Data represent means \pm s.d. ${ }^{*} P<0.05$, NS,

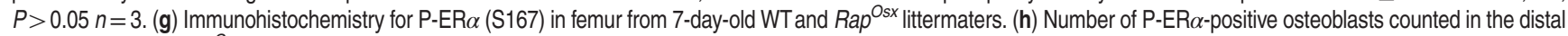
femur of WT and Rap ${ }^{O s x}$ mice. Data represent means \pm s.d. ${ }^{*} P<0.05, n=3$. (i) Effects of CAS6K1, DLX5 and ER $\alpha$ on Runx2 enhancer activity. DLX5 and ER $\alpha$ could synergistically increase the activity of Runx2 enhancer and CAS6K1 could further promote the effects. Data represent means \pm s.d. ${ }^{*} P<0.05, n=3$. (j) Co-immunoprecipitation analysis of ER $\alpha$ and DLX5. Flag-ER $\alpha$ and HA-DLX5 expressing plasmid were co-transfected into 293T cells. After $48 \mathrm{~h}$, immunoprecipitation was performed with whole cell lysate and followed by western blot assay with indicated antibodies. (k) Co-immunoprecipitation analysis of ER $\alpha$ and DLX5 in calvarial osteoblasts. Immunoprecipitation of ER $\alpha$ and lgG was performed with whole cell lysate of calvarial osteoblasts and followed by western blot assay with DLX5 and ER $\alpha$. (I) Co-immunoprecipitation analysis of DLX5 with ER $\alpha$ and phosphorylation site mutant ER $\alpha$. Flag-DLX5 was co-transfected with HA-ER $\alpha$, HA-ER $\alpha$-S171E or HA-ER $\alpha$-S171A respectively in $293 \mathrm{~T}$ cells for $48 \mathrm{~h}$. Immunoprecipitation of Flag and HA was conformed respectively and western blot assay with indicated antibodies. (m) CHIP-qPCR analysis of ER $\alpha$ in Runx2 enhancer region using WT and Rap ${ }^{O s x}$ calvarial osteoblasts. Data represent means \pm s.d. ${ }^{*} P<0.05, n=3$. (n) CHIP-qPCR analysis of DLX5 in Runx2 enhancer region using WT and Rap ${ }^{\text {Osx }}$ calvarial osteoblasts. Data represent means \pm s.d. ${ }^{*} P<0.05, n=3$ 
Immunohistochemical staining was performed following a previously described protocol. ${ }^{37}$ Sections were de-waxed and rehydrated. A solution of $3 \% \mathrm{H}_{2} \mathrm{O}_{2}$ was used to block the activity of endogenous peroxidase. Antigen retrieval was performed with protease $\mathrm{K}$ at $37^{\circ} \mathrm{C}$ for $15 \mathrm{~min}$. Antibodies to Runx2 (1:200, Santa Cruz Biotechnology, Santa Cruz, CA, USA) and P-S6K1 (T389, 1:200, Merck Millipore, Darmstadt, Germany), P-ER $\alpha$ (S167, 1:200, ABclonal, Boston, MA, USA ) were added and incubated overnight at $4{ }^{\circ} \mathrm{C}$. Corresponding biotinylated secondary antibodies were then added and incubated for $1 \mathrm{~h}$ at room temperature, followed by color development with an ABC kit (Vector Labs, Peterborough, UK). We counted the number of Runx2-, P-S6K1- and P-ER $\alpha$-positive cells along the trabecular bone of the distal femur excluding the area within $0.25 \mathrm{~mm}$ from the growth plate.

In situ hybridization was performed as previously described. ${ }^{37}$ Briefly, DIG-labeled RNA probes were used to detect mRNA expression in femurs of 7-day-old $m T O R^{O S x}$, $R_{a p}{ }^{O s x}$ and corresponding WT littermates. Probes used in this study: probes for mouse Col1 $\alpha 1$ (nucleotides 4466-4783, NM_007742, subcloned in pBlueScript), probes for mouse Ocn (nucleotides 39-342, NM_007541, subcloned in pBlueScript). After hybridization, probes were visualized by anti-DIG biotin-conjugated antibody and an ABC kit (Vector Labs). Then, samples were counterstained with hematoxylin. We counted Col1 1 1-positive cells along trabecular bone of the distal femur excluding the area within $0.25 \mathrm{~mm}$ from the growth plate and Ocn-positive cells along cortical bone of the distal femur.

Cell culture. Four-week-old Raptor ${ }^{f / f l}$ mice were killed and the hindlimbs were collected. Bone marrow cells were washed out of the long bones and centrifuged at $500 \times g$ for $10 \mathrm{~min}$. The collected BMSCs were cultured in $\alpha$-MEM with $10 \%$ fetal calf serum and $1 \%$ penicillin/streptomycin. After 14 days, BMSCs were reseeded at $2.5 \times 10^{5} / \mathrm{cm}^{2}$. Twenty-four hours later, BMSCs were infected with adenovirus expressing either CRE recombinase or GFP at a MOI of 10. Then, BMSCs were cultured in osteogenic medium ( $\alpha$-MEM with $10 \%$ FBS and $1 \%$ penicillin/ streptomycin, $100 \mathrm{nM}$ dexamethasone, $50 \mu \mathrm{M}$ L-ascorbic acid, and $10 \mathrm{~mm} \beta$ glycerophosphate) until required.

Parietal bones of P5 mice (mTOR ${ }^{O s x}$, Rap ${ }^{O s x}$ and WT) were digested in $1 \mathrm{mg} / \mathrm{ml}$ collagenase (Sigma) and $2 \mathrm{mg} / \mathrm{ml}$ Dispase II (Sigma) in $\alpha$-MEM for $5 \mathrm{~min}$, three times. The released calvarial cells were cultured in $\alpha$-MEM with 10\% FBS and $1 \%$ penicillin/streptomycin. After 7 days, calvarial cells were reseeded at $5 \times 10^{4} / \mathrm{cm}^{2}$ and cultured in osteogenic medium. For overexpression of CAS6K1 and Runx2, Rap ${ }^{\text {sx }}$ calvarial cells were infected with lentivirus expressing CAS6K1 or Runx2. At the same time WTand Rap ${ }^{\text {Osx }}$ calvarial cells infected with lentivirus expressing GFP were used as control groups. Then these cells were cultured in osteogenic medium.

Alkaline phosphatase staining and alizarin red staining. ALP assay was performed after 7 days of osteoblast differentiation according to the specification of the manufacturer (Beyotime Institute of Biotechnology, Shanghai, China). Mineralized nodule formation was detected by Alizarin red staining 14 days after osteoblast differentiation (Cyagen Biosciences, Santa Clara, CA, USA).

Quantitative PCR (qPCR). Total RNA was extracted from osteoblasts differentiated from BMSCs after 7 days and from calvarial cells using TRIzol, following a standard protocol (Invitrogen, Carlsbad, CA, USA). An aliquot of $500 \mathrm{ng}$ RNA was reverse-transcribed to CDNA using TaKaRa PrimeScript Reverse Transcriptase (TaKaRa Bio Inc., Shiga, Japan). qPCR was performed using a SYBR green mixture (TaKaRa) to detect expression of osteogenic genes. Primers used in this study: Hprt-F:5'-GTTAAGCAGTACAGCCCCAAA-3', Hprt-R: 5'-AGGG CATATCCAACAACAAACTT-3', $\beta$-actin-F: $5^{\prime}$-GTGACGTTGACATCCGTAAAGA-3', $\beta$-actin-R: 5'-GCCGGACTCATCGTACTCC-3', Runx2-F: 5'-CCAACCGAGTCATTT AAGGCT-3', Runx2-R: 5'-GCTCACGTCGCTCATCTTG-3', Col1 $\alpha 1$-F: 5'-GCTCC TCTTAGGGGCCACT-3', Col1 $\alpha 1$-R: 5'-CCACGTCTCACCATTGGGG-3', Ocn-F: CTTGGTGCACACCTAGCAGA-3', Ocn-R: 5'- CTCCCTCATGTGTTGTCCCT-3',

Plasmids. cDNA of S6K1, ER $\alpha$ and DLX5 were cloned into a phage-based plasmid. Constitutively active S6K1 was constructed following previously described methods by converting Thr ${ }^{390}$ to glutamic acid. ${ }^{38}$ Non-phosphorylatable ER $\alpha$ was constructed by converting Ser ${ }^{171}$ into alanine and constitutively phosphorylated ER $\alpha$ was constructed by converting Ser ${ }^{171}$ into glutamic acid. A 3X 89 bp Runx2 enhancer corn sequence was synthesized (by Genescript, Piscataway, NJ, USA) and cloned into a phage-based luciferase reporter. ${ }^{23}$ The Runx2 plasmid was a gift from Dr. Gerard Karsenty's lab. The Runx2 promoter-driven pGL3-based luciferase reporter was kindly provided by Dr. Zhang Feng. ${ }^{39}$
Transient transfection and luciferase reporter assay. Mouse MSC C3H10T1/2 cells were seeded at $4 \times 10^{4}$ cells/well into a 12-well plate, allowed to settle overnight, then transfected using PEI with a luciferase reporter plasmid and Renilla luciferase (Promega, Madison, WI, USA) along with various combinations of expression plasmids as indicated. Empty expression vector plasmids were used as required to make up the total amount of transfected DNA. At $48 \mathrm{~h}$ post-transfection, the cells were lysed with lysis buffer ( $1 \% \mathrm{NP}-40,10 \%$ glycerol, $135 \mathrm{mM} \mathrm{NaCl}$, $20 \mathrm{mM}$ Tris, $\mathrm{pH}$ 8.0) with protease inhibitor and lysates were used for the dualluciferase reporter assay (Promega). In our study, promoter activity was expressed as relative light units normalized to the activity of cotransfected Renilla luciferase.

Co-immunoprecipitation and western blot. For western blot analysis of parietal bone, total proteins were obtained from PO mice with SDS buffer (TaKara). For western blot analysis of BMSCs and calvarial cells, total proteins were obtained from BMSCs and calvarial cells after 7 days of differentiation in osteogenic medium. Proteins ( $60 \mu \mathrm{g}$ of parietal bone protein or $30 \mu \mathrm{g}$ of cells) were separated by $10 \%$ SDS-PAGE followed by western blotting according to a standard protocol. Antibodies used were: mTOR (Cell Signaling Technology, Danvers, MA, USA), Raptor (Cell Signaling Technology), P-S6K1 (T389, Merck Millipore), S6K1 (Cell Signaling Technology), P-S6 (S235/236, Cell Signaling Technology), S6 (Cell Signaling Technology), Runx2 (Santa Cruz Biotechnology), $\beta$-actin (Santa Cruz Biotechnology).

ColP was performed following a method previously described. ${ }^{37}$ Briefly, 293T cells were seeded into a $10 \mathrm{~cm}$ dish at a concentration of $3 \times 10^{6}$ cells/dish and allowed to settle overnight. At $48 \mathrm{~h}$ post-transfection with PEl, cells were lysed and whole cell lysates were used for immunoprecipitation by Flag or HA antibody (Sigma) at $4{ }^{\circ} \mathrm{C}$ overnight. Western blot assay was performed with the indicated antibodies (anti-HA and anti-Flag, Sigma). For ColP analysis of ER $\alpha$ and DLX5 in calvarial osteoblast, whole cell lysates were incubated with IgG and ER $\alpha$ (Sangon Bioteck, Shanghai, China) at $4{ }^{\circ} \mathrm{C}$ overnight. Western blot assay was performed with DLX5 (Abcam, Cambridge, UK) and anti-ER $\alpha$ (Abcam).

Chromatin immunoprecipitation (ChIP) and qPCR. CHIP analysis in WT and Rap ${ }^{O s x}$ calvarial osteoblast was performed following by an Enzymatic Chromatin Immunoprecipitation kit (Cell Signaling Technology) following the instructions of the manufacturer. Briefly, calvarial osteoblasts were cross-linked with $1 \%$ formaldehyde for $10 \mathrm{~min}$ at room temperature followed by quenched with glycine. Chromatin digestion was performed to obtain DNA fragments from $150 \mathrm{bp}$ to 900 bp by Micrococcal Nuclease. Immunoprecipitation was performed with DLX5 (Abcam) and ER $\alpha$ (Abcam), and IgG was used as a negative control. Precipitated DNA was detected by qPCR with specific primers. Primers for Runx2 enhancer: F:5'-CTGCTTTAGGTAGAGGGCTT-3', R: 5'-AATCAGAGTGGAGTCTCAGC-3'.

Statistical analysis. All quantitative data are presented as mean \pm s.d. from at least three independent samples. Student's $t$-test was used for statistical evaluations of two group comparisons. Statistical analysis with more than two groups was performed with one-way analysis of variance (ANOVA). $P<0.05$ was considered statistically significant.

\section{Conflict of Interest}

The authors declare no conflict of interest.

Acknowledgements. The authors thank Drs Gerard Karsenty, Feng Zhang, Minghan Tong for kindly providing reagents. We thank the members of the Zou lab for useful discussions. This work was supported in part by grants from 973 Program from the Chinese Ministry of Science and Technology (MOST) (2014CB964704 and 2015CB964503), the Science and Technology Commission of Shanghai (124119b0101), the National Natural Science Foundation of China (NSFC) (31371463, 31501170, 81371121 and 81570950), the Strategic Priority Research Program of the Chinese Academy of Sciences, Grant No. XDB19000000, Shanghai Summit \& Plateau Disciplines, the 'Chen Xing' project from Shanghai Jiaotong University and WZ is a scholar of the 'National 1000 Young Talents Program of China' and 'the National Science Fund for Excellent Young Scholars' (NSFC) (81322027).

\section{Author contributions}

Conceived and designed the experiments: JW, WZ. Performed the experiments: QD, ZX, NN, XM, SZ, FX. Analyzed the data: QD, WZ. Contributed reagents/materials/ analysis tools: $X Z$, LJ. Wrote the paper: $Q D, W Z$. 
1. Harada S, Rodan GA. Control of osteoblast function and regulation of bone mass. Nature 2003; 423: 349-355.

2. Guntur AR, Rosen CJ. The skeleton: a multi-functional complex organ. New insights into osteoblasts and their role in bone formation: the central role of PI3Kinase. J Endocrinol 2011; 211: $123-130$.

3. Ducy P, Zhang R, Geoffroy V, Ridall AL, Karsenty G. Osf2/Cbfa1: a transcriptional activator of osteoblast differentiation. Cell 1997; 89: 747-754.

4. Otto F, Thornell AP, Crompton T, Denzel A, Gilmour KC, Rosewell IR et al. Cbfa1, a candidate gene for cleidocranial dysplasia syndrome, is essential for osteoblast differentiation and bone development. Cell 1997; 89: 765-771.

5. Komori T, Yagi H, Nomura S, Yamaguchi A, Sasaki K, Deguchi K et al. Targeted disruption of Cbfa1 results in a complete lack of bone formation owing to maturational arrest of osteoblasts. Cell 1997; 89: 755-764.

6. Mundlos S, Otto F, Mundlos C, Mulliken JB, Aylsworth AS, Albright S et al. Mutations involving the transcription factor CBFA1 cause cleidocranial dysplasia. Cell 1997; 89: 773-779.

7. Bhaskar PT, Hay N. The two TORCs and Akt. Dev cell 2007; 12: 487-502.

8. Nojima H, Tokunaga C, Eguchi S, Oshiro N, Hidayat S, Yoshino K et al. The mammalian target of rapamycin (mTOR) partner, raptor, binds the mTOR substrates p70 S6 kinase and 4E-BP1 through their TOR signaling (TOS) motif. J biol chem 2003; 278: 15461-15464.

9. Yamnik RL, Digilova A, Davis DC, Brodt ZN, Murphy CJ, Holz MK. S6 kinase 1 regulates estrogen receptor alpha in control of breast cancer cell proliferation. $J$ biol chem 2009; 284: 6361-6369.

10. Carnevalli LS, Masuda K, Frigerio F, Le Bacquer O, Um SH, Gandin V et al. S6K1 plays a critical role in early adipocyte differentiation. Dev cell 2010; 18: 763-774.

11. Zoncu R, Efeyan A, Sabatini DM. mTOR: from growth signal integration to cancer, diabetes and ageing. Nat rev Mol cell biol 2011; 12: 21-35.

12. Xiang $X$, Zhao J, Xu G, Li Y, Zhang W. mTOR and the differentiation of mesenchymal stem cells. Acta Biochim Biophys Sin (Shanghai) 2011; 43: 501-510.

13. Singha UK, Jiang Y, Yu S, Luo M, Lu Y, Zhang J et al. Rapamycin inhibits osteoblast proliferation and differentiation in MC3T3-E1 cells and primary mouse bone marrow stromal cells. J Cell Biochem 2008; 103: 434-446.

14. Yeh LC, Ma X, Ford JJ, Adamo ML, Lee JC. Rapamycin inhibits BMP-7-induced osteogenic and lipogenic marker expressions in fetal rat calvarial cells. J Cell Biochem 2013; 114: 1760-1771.

15. Lee KW, Yook JY, Son MY, Kim MJ, Koo DB, Han YM et al. Rapamycin promotes the osteoblastic differentiation of human embryonic stem cells by blocking the mTOR pathway and stimulating the BMP/Smad pathway. Stem cells dev 2010; 19: 557-568.

16. Fang $F$, Sun $S$, Wang L, Guan JL, Giovannini M, Zhu Y et al. Neural crest-specific TSC1 deletion in mice leads to sclerotic craniofacial bone lesion. $J$ bone miner res $2015 ; 30$ : 1195-1205.

17. Riddle RC, Frey JL, Tomlinson RE, Ferron M, Li Y, DiGirolamo DJ et al. Tsc2 is a molecular checkpoint controlling osteoblast development and glucose homeostasis. Mol cell biol 2014; 34: 1850-1862.

18. Huang B, Wang Y, Wang W, Chen J, Lai P, Liu Z et al. mTORC1 prevents preosteoblast differentiation through the notch signaling pathway. PLoS genet 2015; 11: e1005426.

19. Chen C, Akiyama K, Wang D, Xu X, Li B, Moshaverinia A et al. mTOR inhibition rescues osteopenia in mice with systemic sclerosis. J Exp Med 2014; 212: 73-91.

20. Chen J, Long F. mTORC1 signaling controls mammalian skeletal growth through stimulation of protein synthesis. Development 2014; 141: 2848-2854.

21. Chen J, Long F. mTORC1 signaling promotes osteoblast differentiation from preosteoblasts. PloS one 2015; 10: e0130627.

22. Martin SK, Fitter S, Dutta AK, Matthews MP, Walkley CR, Hall MN et al. Brief report: the differential roles of $\mathrm{mTORC} 1$ and $\mathrm{mTORC} 2$ in mesenchymal stem cell differentiation. Stem Cells 2015; 33: 1359-1365.

23. Kawane T, Komori H, Liu W, Moriishi T, Miyazaki T, Mori M et al. Dlx5 and mef2 regulate a novel runx2 enhancer for osteoblast-specific expression. J bone miner res 2014; 29: 1960-1969.
24. Yamnik RL, Holz MK. mTOR/S6K1 and MAPK/RSK signaling pathways coordinately regulate estrogen receptor alpha serine 167 phosphorylation. FEBS lett 2010; 584: 124-128.

25. Kammerer M, Gutzwiller S, Stauffer D, Delhon I, Seltenmeyer Y, Fournier B. Estrogen Receptor a (ERa) and Estrogen Related Receptor a (ERRa) are both transcriptional regulators of the Runx2-I isoform. Mol Cell Endocrinol 2013; 369: 150-160.

26. Melville KM, Kelly NH, Khan SA, Schimenti JC, Ross FP, Main RP et al. Female mice lacking estrogen receptor-alpha in osteoblasts have compromised bone mass and strength. $J$ bone miner res 2014; 29: 370-379.

27. Almeida M, lyer S, Martin-Millan M, Bartell SM, Han L, Ambrogini E et al. Estrogen receptoralpha signaling in osteoblast progenitors stimulates cortical bone accrual. J clin invest 2013; 123: 394-404.

28. Guo J-P, Shu S-K, Esposito NN, Coppola D, Koomen JM, Cheng JQ. IKKe phosphorylation of estrogen receptor $\alpha$ Ser-167 and contribution to tamoxifen resistance in breast cancer. $J$ Biol Chem 2010; 285: 3676-3684.

29. Yan B, Zhang Z, Jin D, Cai C, Jia C, Liu W et al. mTORC1 regulates PTHrP to coordinate chondrocyte growth, proliferation and differentiation. Nat commun 2016; 7: 11151.

30. Polak P, Cybulski N, Feige JN, Auwerx J, Ruegg MA, Hall MN. Adipose-specific knockout of raptor results in lean mice with enhanced mitochondrial respiration. Cell metab 2008; 8: 399-410.

31. Lou Y, Javed A, Hussain S, Colby J, Frederick D, Pratap J et al. A Runx2 threshold for the cleidocranial dysplasia phenotype. Hum mol genet 2009; 18: 556-568.

32. Kelly WL, Bryden MM. A modified differential stain for cartilage and bone in whole mount preparations of mammalian fetuses and small vertebrates. Stain technol 1983; 58: 131-134.

33. Yuan $Q$, Jiang $Y$, Zhao $X$, Sato $T$, Densmore M, Schuler $C$ et al. Increased osteopontin contributes to inhibition of bone mineralization in FGF23-deficient mice. $J$ bone miner res 2014; 29: 693-704.

34. Liu W, Zhou L, Zhou C, Zhang S, Jing J, Xie L et al. GDF11 decreases bone mass by stimulating osteoclastogenesis and inhibiting osteoblast differentiation. Nat commun 2016; 7: 12794

35. Guo Y, Sun N, Duan X, Xu X, Zheng L, Seriwatanachai D et al. Estrogen deficiency leads to further bone loss in the mandible of CKD mice. PloS one 2016; 11: e0148804.

36. Andersson GN, Marks S. Tartrate-resistant acid ATPase as a cytochemical marker for osteoclasts. J Histochem Cytochem 1989; 37: 115

37. Zou W, Greenblatt MB, Brady N, Lotinun S, Zhai B, de Rivera $\mathrm{H}$ et al. The microtubuleassociated protein DCAMKL1 regulates osteoblast function via repression of Runx2. J Exp Med 2013; 210: 1793-1806

38. Xia T, Cheng Y, Zhang Q, Xiao F, Liu B, Chen S et al. S6K1 in the central nervous system regulates energy expenditure via MC4R/CRH pathways in response to deprivation of an essential amino acid. Diabetes 2012; 61: 2461-2471.

39. Zhang $F, X u L, X u Q$, Li D, Yang Y, Karsenty G et al. JMJD3 promotes chondrocyte proliferation and hypertrophy during endochondral bone formation in mice. $\mathrm{J} \mathrm{mol} \mathrm{cell} \mathrm{biol}$ 2015; 7: 23-34.

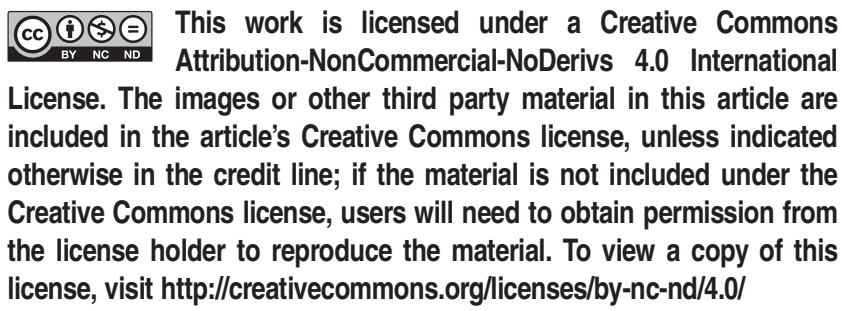

(C) The Author(s) 2017

Supplementary Information accompanies this paper on Cell Death and Differentiation website (http://www.nature.com/cdd) 